THE AstrophysicAL JouRNAL, 540:992-1004, 2000 September 10

(c) 2000. The American Astronomical Society. All rights reserved. Printed in U.S.A.

\title{
COOL COMPANIONS TO HOT WHITE DWARFS
}

\author{
Paul J. GReEN ${ }^{1}$ \\ Harvard-Smithsonian Center for Astrophysics, 60 Garden Street, Cambridge, MA 02138; pgreen@cfa.harvard.edu \\ BABAR Ali \\ Infrared Processing and Analysis Center, Caltech, Mail Code 100-22, 770 S. Wilson Avenue, Pasadena, CA 91125; babar@ipac.caltech.edu \\ AND \\ R. NAPIWOTZKI \\ Dr. Remeis-Sternwarte, Sternwartstr. 7, 96049 Bamberg, Germany; ai23@sternwarte.uni-erlangen.de \\ Received 2000 February 5 ; accepted 2000 April 3
}

\begin{abstract}
Low-mass companions to high-mass stars are difficult to detect, which is partly why the binary fraction for high-mass stars is still poorly constrained. Low-mass companions can be detected more easily however, once high-mass stars turn into white dwarfs. These systems are also interesting as the progenitors of a variety of intensely studied interacting binary systems, like novae, $\mathrm{CVs}$, symbiotics, $\mathrm{Ba}$ and $\mathrm{CH}$ giants, Feige 24-type systems, and dwarf carbon stars. We describe a near-IR photometric search for cool red dwarf companions to hot white dwarfs (WDs). IR photometry offers a sensitive test for low-mass main-sequence (MS) companions. Our sample of EUV-detected WDs offers several advantages over previous (largely proper motion-selected) WD samples: (1) the high WD temperatures $\left(24,000<T_{\text {eff }}<\right.$ $70,000 \mathrm{~K}$ ) insure excellent IR flux contrast with cool dwarfs; (2) the range of evolutionary parameter space occupied by the WDs is considerably narrowed; and (3) the random effects of the intervening ISM provide a complete but reasonably sized sample. While some composite systems have been found optically among WDs detected in recent EUV All-Sky Surveys, we develop an IR technique that probes farther down the main sequence, detecting yet more companions. We use detailed DA model atmosphere fits to optical spectra to predict $K$ magnitudes and distances, against which we contrast our near-IR observations. Our photometric survey reveals 10 DAs with a significant excess in both $J$ and $K$. Half are newly discovered and are most likely previously unrecognized binary systems. Neither the frequency of infrared excess nor the mass estimate of the red dwarf companion correlate with white dwarf mass, as might be expected if either the EUV detectability or mass of the white dwarfs were significantly affected by a companion. Infrared spectra of these systems should help to determine the mass and spectral type of the cool companions presumably causing the IR excess, leading to better estimates of the mass ratio distribution in binaries. Counting previously known binaries, and resolved pairs, we find the total binary fraction of the sample is at least a third. Since most WD progenitors had initial masses $\geq 2 M_{\odot}$, we thus provide a photometric measure of the binary fraction of high-mass stars that would be difficult to perform in high-mass main-sequence stars. We estimate that $90 \%$ of the companions are of type $\mathrm{K}$ or later.
\end{abstract}

Subject headings: binaries: close — stars: atmospheres — stars: evolution — white dwarfs

\section{INTRODUCTION}

EUV-detected stars have revealed in their UV spectra the presence of about 15 hot white dwarf (WD) companions to bright stars in noninteracting binary systems (e.g., Burleigh, Barstow, \& Fleming 1997). These WDs are hidden at optical wavelengths, because of their close proximity to much more luminous companions - main-sequence stars of spectral type K or earlier, or evolved stars.

Many interacting binary systems where the WD is the primary (i.e., optically brightest) star have also been found among EUV-detected systems (e.g., six close, interacting white dwarf/red dwarf binaries by Vennes \& Thorstensen 1994). Optical or ultraviolet spectral observations are most commonly used to detect companions to WD primaries, by searching for (1) the presence of narrow Balmer line emission overlying the broad smooth Balmer absorption of the WD, (2) a composite WD + main-sequence spectrum, or (3) radial velocity $(\mathrm{RV})$ variations. However, only WDs with

\footnotetext{
${ }^{1}$ Visiting Astronomer, Kitt Peak National Observatory, National Optical Astronomy Observatories.
}

very close, or intrinsically active, companions will be found by method (1). For hot WD systems, composite spectra (2) are only expected to be visible if the companion's spectral type is early. RV variations (3) require multiple observations at high spectral resolution, and detection strongly favors close and/or massive companions.

Such discoveries have been strongly dominated by these selection effects, with companions biased to earlier types than predicted by the simulations of deKool \& Ritter (1993) and others. Scaling from the deKool \& Ritter results, Vennes \& Thorstensen (1994) estimate that "at least twice as many close binary systems remain to be identified from EUV surveys, most of them with a low mass secondary." The resulting sample of binaries known to date therefore must diverge strongly from the intrinsic distribution, in overall normalization, as well as in mass and spectral type of the main-sequence companions.

Large proper motion-selected samples (including those observed in the IR e.g., Probst 1983a, 1983b and Zuckerman \& Becklin 1992), are kinematically biased against luminous (statistically more distant) young, hot WDs and span a bewildering range of temperatures, ages, and metallicities. 
Our EUV-selected sample is instead dominated by freshly minted disk DAs, providing a narrower slice of parameter space for stellar evolution (in the wide systems) or for dynamical evolution (in any new close systems). Objects known or proposed to contain white dwarf (WD) stars in currently or previously interacting binary systems constitute diverse laboratories for the study of stellar evolution. A partial list includes novae, cataclysmic variables, symbiotic stars, $\mathrm{Ba}$ and $\mathrm{CH}$ giants, Feige 24-type systems and dwarf carbon stars (Green \& Margon 1994).

We describe here a near-IR photometric survey for lowmass companions to hot white dwarfs (WDs). By investigating only EUV-detected WDs, we obtain a very reasonably sized but complete sample of young WDs, next to which very late-type dwarf companions can be detected in the near-infrared by searching for a $K$ excess. Many hot white dwarfs $\left(T_{\text {eff }}>24,000 \mathrm{~K}\right.$; Finley et al. 1993) have been detected in the recent EUV all-sky surveys. EUV detection of these hot WDs depends primarily on their temperature, distance, and the intervening Galactic ISM. Our sample of EUV WDs (whose selection we define below) offers excellent flux contrast in the IR relative to optical; cool companions will almost always be brighter in the $K$ band than the hot WDs. IR photometry of hot white dwarfs is nearly nonexistent in the literature.

To know what $K$ magnitude to expect for the WDs, we benefit from constraints on $\log g$, radius, and $T_{\text {eff }}$ derivable from optical spectra for the WDs in our sample, using NLTE model atmosphere fits (Napiwotzki, Green, \& Saffer 1999, hereafter NGS99). The resulting predictions for $K$ magnitudes allow a direct search for any IR excess from a cool companion. In some cases, IR colors also provide a preliminary spectral type for the companion.

\section{SAMPLE SELECTION CRITERIA}

We chose to limit our initial sample to DAs, for which model atmospheres provide the best temperature and mass constraints. We start with 73 known DA white dwarfs in the EUVE bright source list (Malina et al. 1994). We exclude sources at low Galactic latitudes $(|b|<15)$ to avoid crowding problems, and in the South $(\delta<-20)$, yielding a list of 27 DAs. $^{2}$ A similar procedure for DAs listed in the ROSAT Wide Field Camera survey Bright Source Catalogue (Pounds et al. 1993) culls an additional 30 objects. Table 1 lists the full sample of 57, including published data used in this paper. Model atmosphere fit parameters for DAs that are not available in NGS99 are taken from Vennes et al. (1997b) or other references listed in Table 1.

Of the 57 sample objects, we present new IR photometry for 47. We did not obtain new measurements for 10 stars with published sensitive optical spectrophotometry and IR photometry and/or known binaries from optical studies (Feige 24, HZ 43, GD 50, V471 Tau (Vennes, Christian, \& Thorstensen 1998), PG 0824+ 289 (Heber et al. 1993), HD 74389B (Liebert, Bergeron, \& Saffer 1990), REJ 1016-052, REJ 1426 + 500 (Vennes et al. 1997a, 1997b), and IK Peg (Wonnacott, Kellett, \& Stickland 1993). Due to observing constraints (a combination of weather, poor seeing and faint objects, or celestial placement of objects) no photometry was obtained for $0138+252$ (PG $0136+251)$.

\footnotetext{
${ }^{2}$ Emission from 40 Eri was resolved with the Einstein HRI, and likely arise mostly from 40 Eri C, the dMe flare star (Cash et al. 1980), not the white dwarf 40 Eri B.
}

On the other hand, we report new IR data for four objects that fell outside the strict sample definitions just outlined. These include the known binaries REJ $1036+460$ (PG 1033+464), REJ $1629+780$ as well as REJ $0148-253$ (GD 1401, also a known binary), and REJ 0457-280 (MCT 0455-2812), which are outside the sample declination limits.

We note that since the initial sample selection, several relevant discoveries pertaining to sample objects have been made. REJ 0134-160 (GD984) has central Balmer emission components produced by a dMe companion. RE $1440+750$ turns out to be a magnetic DA (Dreizler et al. 1994).

\section{OBSERVATIONS AND DATA REDUCTION}

We obtained $J$ - and $K$-band photometry of the sample objects at the Kitt Peak National Observatory ${ }^{3} 2.1 \mathrm{~m}$ telescope using the Infrared Imager (IRIM; Fowler et al. 1988). The $256 \times 256$ IRIM, completed in 1992, is a $\mathrm{HgCdTe}$ NICMOS 3 array, which at the $\mathrm{f} / 15$ focus of the $2.1 \mathrm{~m}$ yields a $280^{\prime \prime} \times 280^{\prime \prime}$ field of view (plate scale of $1^{\prime \prime} .09 \mathrm{pixel}^{-1}$ ).

Tables 2 and 3 summarize our observing procedure. Table 2 lists the individual observing runs dedicated to this project. Column (1) identifies the run. The universal time (UT) date of the run and the photometric standard stars used during each night are shown in columns (2) and (3). Column (4) identifies the references containing infrared $(J$ and $K$-band) magnitudes for the standard stars, and column (5) contains a short overall subjective description for the night conditions. Table 3 summarizes our observing strategy. Column (1) identifies the program object (given as an REJ nnnn + mmm). Column (2) lists the nights on which the target was observed, and column (3) any exceptions to our standard observing procedure of six dithered images per target, each image consisting of six co-added $10 \mathrm{~s}$ exposures. The exceptions are noted in column (3) of Table 3. The number of exposures depended on the faintness of the object. To minimize any hysteresis effects in pixel sensitivity, during the observations, we varied the direction and distance between the position offsets, and never placed a target at the center of the array. At least once during each night, 10-20 (co-added) dark frames of $10 \mathrm{~s}$. duration were also obtained. These are median-combined to form a single dark frame free of cosmic ray hits. The atmospheric seeing (and hence the FWHM of stars) varied from 1.4-2.5 pixels during the observations.

We reduced our image data using the Image Reduction and Analysis Facility (IRAF) software. For each filter separately, the reduction proceeds as follows. All images are corrected for any detector nonlinearities (R. Joyce 1999, private communication). Next, any bad pixels (as defined by the bad pixel mask; see below) are replaced with the average of the linearly interpolated values from adjacent pixels in both dimensions of the image. Each image is then sky subtracted and divided by the flat field.

The sky frame is produced by median-combining all exposures of the star. This also serves to subtract the dark current, since the dark current is included in the sky frames. The flat-field image is created as a median-combined frame from a large $(\geq 30)$ number of individual images. Individual

\footnotetext{
${ }^{3}$ The Kitt Peak National Observatory is operated by the Association of Universities for Research in Astronomy, Inc. (AURA), under cooperative agreement with the National Science Foundation.
} 
TABLE 1

SAMPLE OF EUV-Selected DA White DWARFS

\begin{tabular}{|c|c|c|c|c|c|c|c|}
\hline REJ & Other Names & $V$ & $T_{\text {eff }}$ & $\log g$ & $M / M_{\odot}$ & References & Notes \\
\hline $0007+331^{\mathrm{a}}$. & GD 2 & 13.85 & 45653 & 7.77 & 0.57 & 1,2 & \\
\hline $0134-160^{\mathrm{a}}$. & GD 984, PHL 1043 & 13.96 & 43722 & 7.70 & 0.54 & $1,2,9$ & $\mathrm{DA}+\mathrm{dM}$ \\
\hline $0138+252$ & PG $0136+251$ & 15.87 & 38964 & 9.00 & 1.22 & 1,1 & Peculiar \\
\hline $0148-253^{a} \ldots \ldots$ & GD 1401 & 14.69 & 25707 & 7.70 & 0.50 & $1,2,6$ & $\mathrm{NS}, \mathrm{DA}+\mathrm{dM}$ \\
\hline $0235+034 \ldots \ldots$ & Feige 24 & 12.56 & 62947 & 7.53 & 0.54 & $1,1,13$ & $\mathrm{DAe}+\mathrm{dM}$ \\
\hline $0237-122^{\mathrm{a}} \ldots \ldots$ & PHL 1400 & 14.92 & 31570 & 8.38 & 0.85 & 1,2 & \\
\hline $0348-005 \ldots \ldots$ & GD 50 & 14.04 & 38881 & 8.97 & 1.17 & 1,2 & \\
\hline $0350+171$ & V471 Tau & 13.65 & 34200 & 8.80 & 1.12 & 1,1 & $\mathrm{DA}+\mathrm{K} 0$ \\
\hline $0427+740^{\mathrm{a}} \ldots \ldots$ & & 15.9 & 47549 & 7.84 & 0.61 & 3,2 & Visual pair, $8^{\prime \prime}$ \\
\hline $0443-034^{\mathrm{a}}$. & & 16.9 & 65140 & 9.12 & 1.29 & 3,4 & \\
\hline $0457-280^{\mathrm{a}} \ldots \ldots$ & MCT $0455-2812$ & 13.95 & 50061 & 7.63 & 0.53 & 1,2 & NS \\
\hline $0512-004^{\mathrm{a}}$. & & 14.2 & 31333 & 7.34 & 0.45 & 3,2 & \\
\hline $0521-102^{\mathrm{a}}$. & & 15.81 & 32775 & 8.53 & 0.94 & 1,2 & \\
\hline $0827+288$ & PG $0824+289$ & 14.22 & 51934 & 8.00 & 0.70 & $1,1,12$ & $\mathrm{DA}+\mathrm{dC}+\mathrm{M} 3 \mathrm{~V}$ \\
\hline $0841+032^{\mathrm{a}}$. & & 14.47 & 37687 & 7.68 & 0.52 & 1,2 & \\
\hline $0845+488 \ldots \ldots$ & HD74389B & 15.5: & 39500 & 8.04 & 0.65 & 7,5 & $\mathrm{DA}+\mathrm{A} 2 \mathrm{~V}$ \\
\hline $0902-040^{\mathrm{a}} \ldots \ldots$ & & 13.19 & 22285 & 7.76 & 0.51 & 1,2 & \\
\hline $0907+505^{\mathrm{a}}$. & PG $0904+511$ & 16.54 & 31791 & 8.04 & 0.66 & 1,2 & \\
\hline $0916-194^{\mathrm{a}}$. & & 17.3 & 56400 & 9.12 & 1.29 & 3,4 & Visual pair, 6".4 \\
\hline $0940+502^{\mathrm{a}}$. & PG $0937+506$ & 16.0 & 35511 & 7.62 & 0.51 & 3,2 & \\
\hline $0957+852^{\mathrm{a}}$. & & 15.4 & 50205 & 8.29 & 0.82 & 3,2 & \\
\hline $1016-052$ & & 14.21 & 53827 & 8.08 & 0.65 & $1,1,4$ & $\mathrm{DA}+\mathrm{dM}$ \\
\hline $1019-140^{\mathrm{a}}$. & & 14.93 & 31100 & 7.85 & 0.57 & 1,2 & \\
\hline $1029+450^{\mathrm{a}}$. & PG $1026+454$ & 16.13 & 35017 & 7.64 & 0.51 & 1,2 & \\
\hline $1032+532^{\mathrm{a}}$. & & 14.45 & 42785 & 7.89 & 0.62 & 1,2 & \\
\hline $1033-114$ & G $162-66$, LTT 3870 & 13.01 & 23741 & 7.79 & 0.52 & 1,2 & \\
\hline $1036+460^{\mathrm{a}} \ldots \ldots$ & GD 123 & 14.34 & 28720 & 7.95 & 0.61 & $1,2,10$ & $\mathrm{DA}+\mathrm{K}$ \\
\hline $1043+490^{\mathrm{a}}$. & & 16.23 & 40461 & 7.87 & 0.60 & $1,2,14$ & Visual pair, $8^{\prime \prime}$ \\
\hline $1043+445^{\mathrm{a}}$. & PG $1040+451$ & 16.94 & 48000 & 7.98 & 0.63 & 3,4 & \\
\hline $1044+574^{\mathrm{a}}$. & PG $1041+580$ & 14.64 & 29878 & 7.73 & 0.52 & 1,2 & \\
\hline $1100+713^{\mathrm{a}}$. & PG $1057+719$ & 14.68 & 40328 & 7.75 & 0.55 & 1,2 & \\
\hline $1112+240^{\mathrm{a}}$. & Ton 61 & 15.77 & 39281 & 7.71 & 0.53 & 1,2 & \\
\hline $1122+434^{\mathrm{a}}$. & PG $1120+439$ & 15.8 & 26152 & 8.23 & 0.75 & 3,2 & \\
\hline $1126+183^{\mathrm{a}}$. & PG $1123+189$ & 14.13 & 52747 & 7.69 & 0.56 & $1,2,10$ & $\mathrm{DA}+\mathrm{dM}$ \\
\hline $1128-025^{\mathrm{a}}$. & PG $1125-026$ & 15.73 & 30227 & 8.15 & 0.71 & 1,2 & \\
\hline $1148+183^{a}$. & PG $1145+188$ & 14.33 & 24717 & 7.84 & 0.55 & 1,2 & \\
\hline $1235+233^{a}$. & PG $1232+238$ & 17.4 & 45639 & 7.77 & 0.57 & 3,2 & \\
\hline $1236+475^{\mathrm{a}}$. & PG $1234+482$ & 14.38 & 55570 & 7.57 & 0.53 & 1,1 & Reclassified DB \\
\hline $1257+220^{\mathrm{a}}$. & GD 153 & 13.38 & 38324 & 7.71 & 0.53 & 2 & \\
\hline $1316+295$ & $\mathrm{HZ} 43$ & 12.99 & 49000 & 7.70 & 0.56 & 1,8 & $\mathrm{DA}+\mathrm{dM}$ \\
\hline $1336+694^{\mathrm{a}}$. & PG $1335+701$ & 15.4 & 29067 & 8.27 & 0.78 & 3,2 & \\
\hline $1426+500$ & & 13.6 & 29800 & 7.98 & 0.60 & $3,4,6$ & $\mathrm{DA}+\mathrm{MVe}$ \\
\hline $1431+370^{\mathrm{a}} \ldots \ldots$ & GD 336 & 15.27 & 33988 & 7.86 & 0.58 & 1,2 & \\
\hline $1440+750^{\mathrm{a}}$. & & 15.32 & 42400 & 8.54 & 0.96 & 3,4 & \\
\hline $1446+632^{\mathrm{a}} \ldots \ldots$ & & 16.4 & 37340 & 7.72 & 0.53 & 3,2 & \\
\hline $1629+780^{\mathrm{a}} \ldots \ldots$ & & 13.03 & 39882 & 7.88 & 0.61 & $1,2,11$ & $\mathrm{DA}+\mathrm{M} 4 \mathrm{~V}$ \\
\hline $1638+350^{\mathrm{a}} \ldots \ldots$ & PG $1636+351$ & 14.83 & 34879 & 7.90 & 0.61 & 1,2 & \\
\hline $1643+411^{\mathrm{a}} \ldots \ldots$ & PG $1642+414$ & 16.2 & 28240 & 8.15 & 0.71 & 3,2 & \\
\hline $1650+403^{\mathrm{a}}$. & & 15.83 & 37698 & 7.90 & 0.61 & 1,2 & \\
\hline $1659+440^{\mathrm{a}} \ldots \ldots$ & PG $1658+440$ & 14.62 & 30510 & 9.36 & 1.31 & 3,5 & Magnetic DA \\
\hline $1711+664^{a} \ldots \ldots$ & & 17.10 & 47994 & 8.78 & 1.09 & 1,2 & Visual pair, 2".5 \\
\hline $1726+583^{a} \ldots \ldots$ & PG $1725+586$ & 15.45 & 52413 & 8.14 & 0.75 & 1,2 & Visual pair, 6".7 \\
\hline $1800+683^{a} \ldots \ldots$ & KUV $18004+6836$ & 14.74 & 43868 & 7.74 & 0.56 & 1,2 & \\
\hline $1820+580^{\mathrm{a}} \ldots \ldots$ & & 13.95 & 43296 & 7.70 & 0.54 & 1,2 & \\
\hline $1845+682^{\mathrm{a}} \ldots \ldots$ & KUV $18453+6819$ & 15.5 & 35599 & 8.15 & 0.72 & 3,2 & \\
\hline $2116+735^{a} \ldots \ldots$ & KUV $21168+7338$ & 15.0 & 49777 & 7.65 & 0.54 & 3,2 & \\
\hline $2126+192 \ldots \ldots$ & IK Peg & 6.07 & $\ldots$ & $\ldots$ & $\ldots$ & $\ldots$ & $\mathrm{DA}+\mathrm{A} 8 \mathrm{~m}$ \\
\hline $2207+252 \ldots \ldots$ & & 14.58 & 26129 & 8.20 & 0.74 & 1,2 & Visual pair, 8".9 \\
\hline $2312+104^{a} \ldots \ldots$ & GD 246 & 13.09 & 51950 & 7.76 & 0.59 & 1,2 & \\
\hline
\end{tabular}

NOTES.-NS means not in official sample, but may have optical fits in NGS99 and/or IR data. Visual pairs within 10" and $\Delta R=3$ mag are noted with separations

${ }^{\text {a }}$ New IR photometry presented in this work.

REFERENCES.-First reference is for $V$ magnitude, the second for $T_{\text {eff }}, \log g$, and $M / M_{\odot}$; the third if listed is for companion's spectral type and/or mass of previously known companion. If a multiple system has no third reference listed, it has several references available in SIMBAD. (1) Marsh et al. 1997; (2) NGS99; (3) $V$ from McCook \& Sion 1999; (4) Vennes et al. 1997b; (5) Schmidt et al. 1992; (6) Finley et al. 1997; (7) SIMBAD; (8) Napiwotski et al. 1993; (9) Vennes et al. 1998; (10) Green et al. 1986; (11) Catalàn et al. 1995; (12) Green \& Margon 1994; (13) Vennes \& Thorstensen 1994; (14) Schwartz et al. 1995. 
TABLE 2

SUMMARY OF OBSERVING RUNS

\begin{tabular}{|c|c|c|c|c|}
\hline $\begin{array}{l}\text { ID } \\
(1)\end{array}$ & $\begin{array}{l}\text { UT Date } \\
(2)\end{array}$ & $\begin{array}{l}\text { Standard Stars } \\
\text { Used }^{\mathrm{a}} \\
\text { (3) }\end{array}$ & $\begin{array}{c}\text { Standard } \\
\text { Stars } \\
\text { References } \\
\text { (4) }\end{array}$ & $\begin{array}{c}\text { Conditions } \\
\text { (5) }\end{array}$ \\
\hline $\mathrm{KP} 01 \ldots \ldots$ & 1996 Jan 04 & FS 6, 10, 19, LHS 254, LHS 325 & 1,2 & Clear or light cirrus. \\
\hline KP02 ...... & 1996 Jan 05 & FS 10, 19, LHS 191, LHS 2347 & 1,2 & Cirrus, but eventually very clear. \\
\hline KP03 ...... & 1996 Jan 06 & FS 2, 10, 19, LHS 2502 & 1,2 & Heavy cirrus. \\
\hline KP04 ...... & 1996 Jan 07 & FS 15, 10, 19, LHS 191 & 1,2 & High thin cirrus. \\
\hline $\mathrm{KP} 05 \ldots \ldots$ & 1996 Jul 02 & $\ldots$ & $\ldots$ & Cloudy, no data. \\
\hline KP06 ...... & 1996 Jul 03 & $\ldots$ & $\ldots$ & Cloudy, no data. \\
\hline KP07 ...... & 1996 Jul 04 & $\ldots$ & $\ldots$ & Cloudy, no data. \\
\hline KP08 ...... & 1996 Jul 05 & $\ldots$ & $\ldots$ & Cloudy, no data. \\
\hline KP09 ...... & 1996 Jul 06 & $\ldots$ & $\ldots$ & Cloudy, no data. \\
\hline $\mathrm{KP} 10 \ldots \ldots$ & 1996 Oct 29 & FS $1,6,9,12,15,29,31$ & 1 & Mostly clear. \\
\hline KP11 ..... & 1996 Oct 30 & FS 1, 10, 14, 29, GJ 1057, GJ 1002 & 1,2 & Photometric. \\
\hline KP12 ....... & 1998 Jan 20 & $\cdots$ & $\cdots$ & Cloudy. \\
\hline $\mathrm{KP} 13 \ldots \ldots$ & 1998 Jan 21 & $\ldots$ & $\ldots$ & Fog. Telescope closed. \\
\hline KP14 ...... & 1998 Jan 22 & FS $2,6,21,24,18,19$ & 1 & Clear. \\
\hline KP15 ...... & 1998 Мay 09 & FS 21, 23, 24, 27, 29, 33 & 1 & Clear. \\
\hline KP16...... & 1998 May 10 & FS $16,20,21,23,35$ & 1 & Mostly clear with moderate haze. \\
\hline KP17 ...... & 1998 May 11 & FS 19, 21, 27 & 1 & Hazy \\
\hline
\end{tabular}

${ }^{\text {a }} \mathrm{FS}=$ UKIRT faint star.

REFERENCES.- (1) Casali \& Hawarden 1992; (2) Leggett 1992.

images are median-subtracted to account for sky/ background variations. The flat-field image is dark subtracted and normalized. The flat-field image is also used to produce the bad pixel mask, as defined by the histogram distribution of pixel intensities. All pixels with intensity values near or beyond the extreme values of the histogram distribution are defined to be bad. The bad pixel mask was produced once and used throughout.

Since our sample is defined to be at high Galactic latitude and hence uncrowded, simple aperture photometry is ade-

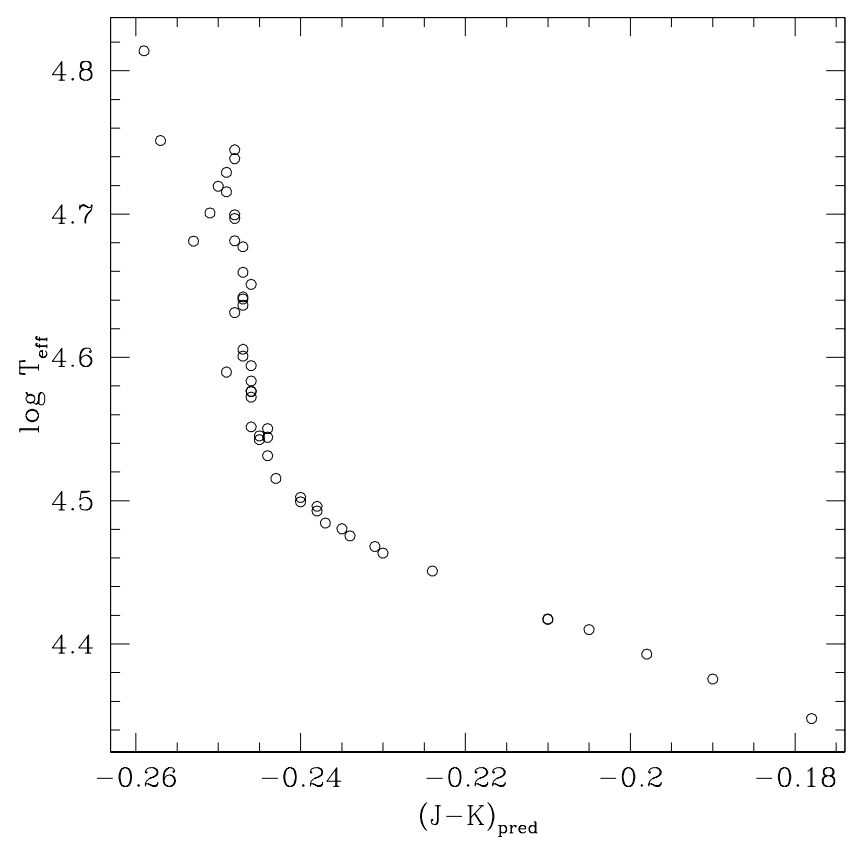

FIG. 1.-Sample DA white dwarf temperature from best-fit model atmospheres of NGS99 plotted against our predicted $(J-K)$ color for the star assuming it is solitary. DA colors are nearly degenerate for temperatures above about $35,000 \mathrm{~K}$. quate. Following the arguments presented in Howell (1989) we used a 4.0 pixel diameter aperture to maximize the signal-to-noise ratio $(\mathrm{S} / \mathrm{N})$. Instrumental magnitudes are measured for each object using the IRAF task QPHOT with the radius of the inner sky annulus set to 10 pixels and the width of sky annulus set to 5 pixels.

For target stars REJ 0443-034, REJ 0907+505, REJ $0940+502$, and REJ $1442+632$, visual inspection failed to reveal the object on individual images. In such cases, we shift (by integer pixels) and combine all the images of the field to form an average mosaicked image. Individual frames are median-subtracted prior to averaging to remove any zero point offsets due to varying sky/background conditions. The pixel shifts are calculated from other nearby stars in the field. The photometry procedure for these mosaicked images is described in the Appendix.

We derive the zero point calibrations for the instrumental magnitudes from photometric standard stars observed at a variety of air masses each night. These standards stars were mostly selected from the UKIRT faint standard list (Casali \& Hawarden 1992). The photometric standards for each night are listed in Table 2.

We eliminated the most extreme outlying measurement for an object if its elimination reveals it to be more than 5 times the root mean square (rms) of the resulting average magnitude. The resulting relative photometric error $\sigma$ is taken to be the rms of all measurements from each individual frame. To obtain the final magnitude error, we add to $\sigma$ the rms dispersion in the standard star zero points taken over the night. The final magnitudes for targets are the mean values produced by averaging the photometry from all nights on which the target was observed. If the target was observed on more than two nights, we use the algorithm described above to eliminate outlying measurements.

Table 4 lists for each object with new IR observations both the observed and predicted magnitudes. Column (1) lists the RE name, (2) the observed $J$ magnitude, and (3) error. Columns (4) and (5) list the $K$ magnitude and error, 
TABLE 3

SUMMARY OF TARGET OBSERVATIONS

\begin{tabular}{|c|c|c|}
\hline Target & Observing Runs & Notes $^{a}$ \\
\hline $0007+331 \ldots \ldots$ & КP02, КР04, КР10 & \\
\hline $0134-160 \ldots \ldots$ & $\begin{array}{l}\text { КP02, КP04, } \\
\text { KP10 }\end{array}$ & \\
\hline $0148-253 \ldots \ldots$ & КР02, КР03, КР04 & \\
\hline $0237-122 \ldots \ldots$ & $\begin{array}{l}\text { KP04, } \\
\text { KP10 }\end{array}$ & kn9 \\
\hline $0348-005 \ldots \ldots$ & $\begin{array}{l}\text { KP01, КP02, КР03, КP04, } \\
\text { КР11 }\end{array}$ & jn $3, \mathrm{kn} 3$ \\
\hline $0427+740 \ldots \ldots$ & $\begin{array}{l}\text { KP01, КР03, КР04, } \\
\text { КР14 }\end{array}$ & kn9 \\
\hline $0443-034 \ldots \ldots$ & $\begin{array}{l}\text { KP03, } \\
\text { KP11 }\end{array}$ & jn9, kn9 \\
\hline $0457-280 \ldots \ldots$ & KP03, KP11 & \\
\hline $0512-004 \ldots \ldots$ & $\begin{array}{l}\text { KР01, КР03, КР04, КР10, } \\
\text { КР14 }\end{array}$ & jn9, kn9 \\
\hline $0521-102 \ldots \ldots$ & $\begin{array}{l}\text { KP01, KP03, } \\
\text { KP10, } \\
\text { KP14 }\end{array}$ & $\begin{array}{l}\text { jn9, kn9 } \\
\text { kn9 }\end{array}$ \\
\hline $0827+284 \ldots \ldots$ & KP11 & \\
\hline $0841+032 \ldots \ldots$ & $\begin{array}{l}\text { KР01, КР10, } \\
\text { КР14 }\end{array}$ & $\begin{array}{l}\text { jn } 9, \text { kn9 } \\
\text { kn9 }\end{array}$ \\
\hline $0902-040 \ldots \ldots$ & $\begin{array}{l}\text { KP01, KP04 } \\
\text { KP10 }\end{array}$ & kn9 \\
\hline $0907+505 \ldots \ldots$ & $\begin{array}{l}\text { KP03, КР04, KР11, КР14, } \\
\text { KP17 }\end{array}$ & $\begin{array}{l}\text { jn9, kn9 } \\
\text { kn18 }\end{array}$ \\
\hline $0916-194 \ldots \ldots$ & KP02 & jn9, kn9 \\
\hline $0940+502 \ldots \ldots$ & KP01, & jn9, kn9 \\
\hline & KP15, & $\mathrm{jc} 1, \mathrm{kc} 1$ \\
\hline & KP16, & $\mathrm{jc} 2, \mathrm{jn} 9, \mathrm{kc} 2, \mathrm{kn} 9$ \\
\hline & KP17 & $\mathrm{kc} 9, \mathrm{kn} 18$ \\
\hline $0957+852 .$. & KP02, & \\
\hline & KP14, & kn9 \\
\hline & KP16, & $\mathrm{jc} 2, \mathrm{jn} 9, \mathrm{kc} 2, \mathrm{kn} 9$ \\
\hline & KP17 & $\mathrm{kc} 9, \mathrm{kn} 9$ \\
\hline $1019-140 \ldots \ldots$ & KP02 & \\
\hline $1029+450 \ldots \ldots$ & КP02 & \\
\hline $1032+532 \ldots \ldots$ & $\begin{array}{l}\text { KP01, KP04, } \\
\text { KP14 }\end{array}$ & kn9 \\
\hline $1033-114 \ldots \ldots$ & КР01, КР02, КР03, КР04 & \\
\hline $1036+460 \ldots \ldots$ & KP02 & \\
\hline $1040+451 \ldots \ldots$ & KP15, & $\mathrm{jc} 1, \mathrm{jn} 12, \mathrm{kc} 1, \mathrm{kn} 12$ \\
\hline & KP16, & jc2, jn9, kc2, kn9 \\
\hline & KP17 & $\mathrm{kc} 12$ \\
\hline $1043+490 \ldots \ldots$ & KP02, & \\
\hline & KP14, & kn9 \\
\hline & KP15, & jc1, jn9, kc2, kn9 \\
\hline $1044+574 \ldots \ldots$ & KP02 & \\
\hline $1100+713 \ldots \ldots$ & KP02, & \\
\hline & KP17 & kn15 \\
\hline $1112+240 \ldots \ldots$ & KP16 & $\mathrm{jc} 2, \mathrm{jn} 9, \mathrm{kc} 2, \mathrm{kn} 9$ \\
\hline $1122+434 \ldots \ldots$ & KP02, & \\
\hline & KP16 & $\mathrm{jc} 2, \mathrm{kc} 2, \mathrm{kn} 9$ \\
\hline $1126+183 \ldots \ldots$ & KP01, КР04 & \\
\hline $1128-025 \ldots \ldots$ & $\begin{array}{l}\text { KP02, } \\
\text { KP16 }\end{array}$ & $\mathrm{jc} 1, \mathrm{kc} 1$ \\
\hline $1148+183 \ldots \ldots$ & KP01, КР03 & \\
\hline $1235+233 \ldots \ldots$ & KP14, & kn9 \\
\hline & KP16, & $\mathrm{jc} 2, \mathrm{jn} 9, \mathrm{kc} 3, \mathrm{kn} 9$ \\
\hline & KP17, & $\mathrm{kc} 12, \mathrm{kn} 9$ \\
\hline $1234+482 \ldots \ldots$ & KP14 & kn9 \\
\hline $1257+220 \ldots \ldots$ & КР01, КР02, КР03 & \\
\hline $1336+694 \ldots \ldots$ & КР03, КР04, & \\
\hline & $\begin{array}{l}\text { KP14, } \\
\text { KP16 }\end{array}$ & $\begin{array}{l}\text { kn9 } \\
\text { jc1, jn9, kc2, kn9 }\end{array}$ \\
\hline $1431+370 \ldots \ldots$ & КР03, КР04 & \\
\hline
\end{tabular}

TABLE 3-Continued

\begin{tabular}{|c|c|c|}
\hline Target & Observing Runs & Notes $^{a}$ \\
\hline \multirow[t]{3}{*}{$1431+370 \ldots \ldots$} & KP10, & kn9 \\
\hline & KP16, & kn9,jn9,jc1,kc2 \\
\hline & KP17 & kn9,kc12 \\
\hline \multirow[t]{3}{*}{$1440+750 \ldots \ldots$} & KP14, & \\
\hline & KP16, & $\mathrm{jc} 1, \mathrm{jn} 9, \mathrm{kc} 2, \mathrm{kn} 9$ \\
\hline & KP17 & $\mathrm{kc} 12, \mathrm{kn} 9$ \\
\hline \multirow[t]{3}{*}{$1446+632 \ldots \ldots$} & KP14, & \\
\hline & KP16, & $\mathrm{jc} 2, \mathrm{jn} 9, \mathrm{kc} 3, \mathrm{kn} 15$ \\
\hline & KP17 & $\mathrm{kc} 12, \mathrm{kn} 9$ \\
\hline $1629+780 \ldots \ldots$ & KP15 & $\mathrm{jc} 1, \mathrm{kc1}$ \\
\hline $1638+350 \ldots \ldots$ & KP15 & $\mathrm{jc} 1, \mathrm{kc} 1, \mathrm{kn} 9$ \\
\hline \multirow[t]{3}{*}{$1643+411 \ldots \ldots$} & KP15, & $\mathrm{jc} 1, \mathrm{jn} 9, \mathrm{kc} 2, \mathrm{kn} 9$ \\
\hline & KP16, & $\mathrm{jc} 2, \mathrm{jn} 9, \mathrm{kc} 3, \mathrm{kn} 15$ \\
\hline & KP17 & $\mathrm{kc} 12, \mathrm{kn} 9$ \\
\hline \multirow[t]{2}{*}{$1650+403 \ldots \ldots$} & KP15, & jc1, jn9, kc1, kn9 \\
\hline & KP16 & $\mathrm{jc} 2, \mathrm{jn} 9, \mathrm{kc} 2, \mathrm{kn} 9$ \\
\hline \multirow[t]{4}{*}{$1658+441 \ldots \ldots$} & KP14, & \\
\hline & KP15, & jc1, jn9, kc1, kn9 \\
\hline & KP16, & $\mathrm{jc} 2, \mathrm{kn} 9, \mathrm{kc} 2, \mathrm{kn} 9$ \\
\hline & KP17 & $\mathrm{kc} 12, \mathrm{kn} 9$ \\
\hline $1711+664 \ldots \ldots$ & KP15 & $\mathrm{jc} 1, \mathrm{jn} 18, \mathrm{kc} 1, \mathrm{kn} 9$ \\
\hline $1726+583 \ldots \ldots$ & KP15 & $\mathrm{jc} 1, \mathrm{jn} 9, \mathrm{kc} 1, \mathrm{kn} 9$ \\
\hline \multirow[t]{3}{*}{$1800+683 \ldots \ldots$} & KP15, & jc1, jn9, kc1, kn9 \\
\hline & KP16, & $\mathrm{jc} 2, \mathrm{jn} 9, \mathrm{kc} 2, \mathrm{kn} 9$ \\
\hline & KP17, & $\mathrm{kc} 12, \mathrm{kn} 9$ \\
\hline \multirow[t]{2}{*}{$1820+580 \ldots \ldots$} & KP11, & \\
\hline & KP15 & $\mathrm{jc} 1, \mathrm{jn} 9, \mathrm{kc} 1, \mathrm{kn} 9$ \\
\hline \multirow{2}{*}{$1845+682 \ldots \ldots$} & KP11, & \\
\hline & KP15 & jc1, jn9, kc1, kn9 \\
\hline \multirow[t]{4}{*}{$2116+735 \ldots \ldots$} & KP10, KP11, & \\
\hline & KP15, & jc1, jn9, kc1, kn9 \\
\hline & KP16, & $\mathrm{jc} 2, \mathrm{jn} 9, \mathrm{kc} 3, \mathrm{kn} 9$ \\
\hline & KP17 & $\mathrm{kc12}, \mathrm{kn} 9$ \\
\hline $2127-221 \ldots \ldots$ & KP10 & jn9, kn9 \\
\hline \multirow[t]{2}{*}{$2207+252 \ldots \ldots$} & KP15, & $\mathrm{jc} 1, \mathrm{jn} 9, \mathrm{kc} 1, \mathrm{kn} 9$ \\
\hline & KP17 & \\
\hline $2312+104 \ldots \ldots$ & КР02, КР03, КР04 & \\
\hline $2353-243 \ldots \ldots$ & KP10 & jn9, kn9 \\
\hline
\end{tabular}

NoTES.- " $X \mathrm{c} Y$ " denotes that in the $X$ bandpass the number of co-adds performed per exposure is $Y$ (e.g., jc1 means 1 co-add per exposure in $J$ band). " $X \mathrm{n} Y$ " denotes that in the $X$ bandpass the number of combined dithered exposures is $Y$ (e.g., kn9 means 9 dithered exposures combined in the $K$ band).

${ }^{\text {a }}$ The number of $10 \mathrm{~s}$ co-adds per exposure in both $J$ and $K$ bands is nominally six, and the number of dithered exposures is also six, except as noted in the last column.

respectively, with the $(J-K)$ color and error in columns (6) and (7). The absolute magnitude $M_{V}$ from the best-fit spectral model is shown in column (8). We list in column (9) the predicted $(V-K)$ color based on synthetic photometry of the best-fit DA models. Predicted near-IR magnitudes for the DA WDs are listed in columns (10)-(11), and notes in column (12). We note that our predictions of near-IR colors for these systems have very little scatter over the full sample range, with mean $(J-K)=-0.24 \pm 0.02$, as can be seen from Figure 1. DA colors - even $(V-K)$-are nearly degenerate for DA temperatures higher than about $35,000 \mathrm{~K}$.

\section{ANALYSIS}

4.1. Predicting Near-Infrared Magnitudes and Colors

We use a grid of 184 NLTE model spectra for DA WDs, with $T_{\text {eff }}$ from 20,000 to 100,000 (in 16 steps, coarser toward 
TABLE 4

Observed and Predicted Magnitudes and Colors of DA White Dwarf Stars

\begin{tabular}{|c|c|c|c|c|c|c|c|c|c|c|c|}
\hline \multirow[b]{2}{*}{$\begin{array}{c}\text { RE NAME } \\
\text { (1) }\end{array}$} & \multicolumn{6}{|c|}{ OBSERVED } & \multicolumn{4}{|c|}{ PREDICTED } & \multirow[b]{2}{*}{$\begin{array}{c}\text { Notes } \\
(12)\end{array}$} \\
\hline & $\begin{array}{r}J \\
(2)\end{array}$ & $\begin{array}{l}\sigma_{J} \\
(3)\end{array}$ & $\begin{array}{l}K \\
(4)\end{array}$ & $\begin{array}{l}\sigma_{K} \\
(5)\end{array}$ & $\begin{array}{l}(J-K) \\
(6)\end{array}$ & $\begin{array}{c}\sigma_{(J-K)} \\
(7)\end{array}$ & $\begin{array}{l}M_{V} \\
(8)\end{array}$ & $\begin{array}{c}(V-K)_{p} \\
\quad(9)\end{array}$ & $\begin{array}{c}J_{p} \\
(10)\end{array}$ & $\begin{array}{c}K_{p} \\
(11)\end{array}$ & \\
\hline $0007+331$ & 14.62 & 0.02 & 14.82 & 0.07 & -0.21 & 0.07 & 9.06 & -0.72 & 14.33 & 14.57 & \\
\hline $0134-160$ & 12.95 & 0.01 & 12.21 & 0.01 & 0.74 & 0.01 & 9.00 & -0.72 & 14.43 & 14.68 & \\
\hline $0148-253$ & 12.33 & 0.01 & 11.56 & 0.04 & 0.76 & 0.04 & 9.98 & -0.60 & 15.08 & 15.29 & \\
\hline $0237-122$ & 15.74 & 0.06 & 15.93 & 0.12 & -0.18 & 0.14 & 10.68 & -0.68 & 15.36 & 15.60 & \\
\hline $0348-005$ & 14.80 & 0.06 & 14.66 & 0.52 & 0.14 & 0.52 & 11.46 & -0.71 & 14.50 & 14.75 & 1 \\
\hline $0427+740$ & 15.64 & 0.03 & 15.62 & 0.12 & 0.02 & 0.12 & 9.13 & -0.73 & 16.38 & 16.63 & \\
\hline $0443-034$ & 17.38 & 0.05 & 17.99 & 0.07 & -0.61 & 0.08 & 11.23 & -0.75 & 16.49 & 16.75 & \\
\hline $0457-280$ & 14.62 & 0.02 & 14.80 & 0.03 & -0.18 & 0.03 & 8.69 & -0.73 & 14.43 & 14.68 & \\
\hline $0512-004$ & 14.43 & 0.03 & 14.67 & 0.01 & 0.15 & 0.40 & 8.81 & -0.67 & 14.63 & 14.87 & \\
\hline $0521-102$ & 16.76 & 0.20 & 17.13 & 0.13 & -0.37 & 0.24 & 10.87 & -0.69 & 16.26 & 16.50 & \\
\hline $0550-240$ & 16.93 & 0.10 & 17.28 & 0.41 & -0.34 & 0.42 & 9.03 & -0.73 & 17.18 & 17.43 & \\
\hline $0841+032$ & 15.17 & 0.02 & 15.49 & 0.01 & -0.33 & 0.02 & 9.17 & -0.70 & 14.93 & 15.18 & \\
\hline $0902-040$ & 13.70 & 0.01 & 13.90 & 0.05 & -0.20 & 0.05 & 10.37 & -0.53 & 13.54 & 13.72 & \\
\hline $0907+505$ & 17.16 & 0.10 & 17.54 & 0.64 & -0.39 & 0.65 & 10.09 & -0.68 & 16.98 & 17.22 & \\
\hline 0916-194 & 13.94 & 0.02 & 13.64 & 0.04 & 0.36 & 0.10 & 11.36 & -0.74 & 17.78 & 18.04 & \\
\hline $0940+502$ & 16.73 & 0.07 & 16.97 & 0.02 & -0.24 & 0.07 & 9.14 & -0.70 & 16.45 & 16.70 & \\
\hline $0957+852$ & 16.49 & 0.08 & 16.72 & 0.09 & -0.23 & 0.12 & 9.87 & -0.73 & 15.88 & 16.13 & \\
\hline $1019-140$ & 15.17 & 0.04 & 15.45 & 0.14 & -0.28 & 0.14 & 9.82 & -0.67 & 15.36 & 15.60 & \\
\hline $1029+450$ & 16.92 & 0.09 & 17.54 & 0.70 & -0.62 & 0.71 & 9.20 & -0.69 & 16.58 & 16.82 & \\
\hline $1032+532$ & 15.38 & 0.01 & 15.45 & 0.11 & -0.07 & 0.11 & 9.35 & -0.72 & 14.92 & 15.17 & \\
\hline $1033-114$ & 14.80 & 0.06 & 14.66 & 0.52 & 0.14 & 0.52 & 10.31 & -0.56 & 13.38 & 13.57 & \\
\hline $1036+460$ & 12.62 & 0.04 & 11.89 & 0.09 & 0.72 & 0.10 & 9.95 & -0.65 & 14.76 & 14.99 & \\
\hline $1043+490$ & 16.86 & 0.02 & 16.95 & 0.22 & -0.10 & 0.22 & 8.40 & -0.72 & 16.71 & 16.95 & \\
\hline $1043+445$ & 18.00 & 0.05 & 17.48 & 0.09 & 0.52 & 0.11 & 9.43 & -0.73 & 17.42 & 17.67 & \\
\hline $1044+574$ & 15.32 & 0.04 & 15.73 & 0.17 & -0.42 & 0.18 & 9.72 & -0.66 & 15.06 & 15.30 & \\
\hline $1100+713$ & 15.51 & 0.10 & 15.78 & 0.17 & -0.27 & 0.20 & 9.19 & -0.71 & 15.14 & 15.39 & \\
\hline $1112+240$ & 16.37 & 0.21 & 16.51 & 0.55 & -0.14 & 0.59 & 9.17 & -0.71 & 16.24 & 16.48 & \\
\hline $1122+434$ & 16.12 & 0.05 & 16.39 & 0.34 & -0.27 & 0.34 & 10.82 & -0.61 & 16.20 & 16.41 & \\
\hline $1126+183$ & 12.74 & 0.09 & 12.04 & 0.10 & 0.70 & 0.14 & 8.47 & -0.73 & 14.61 & 14.86 & \\
\hline $1128-025$ & 16.18 & 0.06 & 16.48 & 0.17 & -0.30 & 0.18 & 10.38 & -0.67 & 16.16 & 16.40 & \\
\hline $1148+183$ & 14.96 & 0.06 & 15.22 & 0.20 & -0.26 & 0.20 & 10.31 & -0.58 & 14.71 & 14.91 & \\
\hline $1235+233$ & 18.31 & 0.11 & $\ldots$ & $\ldots$ & -2.36 & 0.28 & 9.06 & -0.72 & 17.88 & 18.12 & 2 \\
\hline $1236+475$ & 14.91 & 0.06 & 14.90 & 0.16 & 0.01 & 0.17 & 8.37 & -0.73 & 14.86 & 15.11 & \\
\hline $1257+220$ & 14.17 & 0.06 & 14.43 & 0.02 & -0.26 & 0.05 & 9.21 & -0.71 & 13.84 & 14.09 & \\
\hline $1336+694$ & 15.85 & 0.02 & 16.12 & 0.04 & -0.28 & 0.04 & 10.66 & -0.65 & 15.82 & 16.05 & \\
\hline $1431+370$ & 15.97 & 0.04 & 16.37 & 0.16 & -0.40 & 0.17 & 9.65 & -0.69 & 15.72 & 15.97 & \\
\hline $1440+750$ & 17.21 & 0.11 & 16.64 & 0.12 & 0.57 & 0.16 & 11.39 & -0.70 & 15.77 & 16.02 & \\
\hline $1446+632$ & 16.77 & 0.11 & 17.77 & 0.07 & -1.00 & 0.12 & 9.26 & -0.70 & 16.86 & 17.10 & \\
\hline $1629+780$ & 10.96 & 0.03 & 11.19 & 0.04 & -0.23 & 0.05 & 9.42 & -0.71 & 13.49 & 13.74 & \\
\hline $1638+350$ & 15.55 & 0.11 & 15.57 & 0.64 & -0.01 & 0.64 & 9.67 & -0.70 & 15.28 & 15.53 & \\
\hline $1643+411$ & 16.86 & 0.10 & 17.46 & 0.05 & -0.61 & 0.11 & 10.53 & -0.64 & 16.62 & 16.84 & \\
\hline $1650+403$ & 16.60 & 0.08 & 16.86 & 1.05 & -0.26 & 1.05 & 9.54 & -0.71 & 16.29 & 16.54 & \\
\hline $1658+441$ & 15.47 & 0.10 & 15.68 & 0.02 & -0.21 & 0.10 & 12.70 & -0.67 & 15.06 & 15.29 & \\
\hline $1711+664$ & 15.02 & 0.01 & 14.19 & 0.18 & 0.83 & 0.18 & 10.84 & -0.73 & 17.58 & 17.83 & \\
\hline $1726+583$ & 16.40 & 0.26 & 16.17 & 1.51 & 0.23 & 1.53 & 9.56 & -0.73 & 15.93 & 16.18 & \\
\hline $1800+683$ & 15.54 & 0.04 & 15.87 & 0.04 & -0.33 & 0.06 & 9.06 & -0.72 & 15.21 & 15.46 & \\
\hline $1820+580$ & 14.07 & 0.04 & 13.42 & 0.01 & 0.65 & 0.04 & 9.01 & -0.72 & 14.42 & 14.67 & \\
\hline $1845+682$ & 14.85 & 0.10 & 14.37 & 0.19 & 0.47 & 0.22 & 10.06 & -0.70 & 15.95 & 16.20 & \\
\hline $2116+735$ & 15.54 & 0.01 & 15.93 & 0.06 & -0.39 & 0.06 & 8.74 & -0.73 & 15.48 & 15.73 & \\
\hline $2207+252$ & 14.34 & 0.08 & 14.06 & 0.22 & 0.28 & 0.23 & 10.77 & -0.61 & 14.98 & 15.19 & 3 \\
\hline $2312+104$ & 13.81 & 0.04 & 14.06 & 0.01 & -0.14 & 0.75 & 8.88 & -0.73 & 13.57 & 13.82 & \\
\hline
\end{tabular}

NoTES.-Objects with significant IR excess, are shown in boldface. (1) Faint IR standard star photometry from Casali \& Hawarden 1992. (2) Object is barely discernible on $K$ band image; $K$ photometry unreliable. (3) IR photometry known to include nearby optically resolved object at 2.2 .

the high end) and $\log g$, in 12 equal steps from 7.0 to 9.75 . We predict colors or magnitudes for each grid model by convolving the model spectrum over the observed filter bandpasses. In the visual, we use Mathews \& Sandage $V$ filter curves and typical CCD quantum efficiency (QE) curves, while in the near-IR, we use the actual NOAO filter transmission curves for the $J$ and $K$ filters, along with the IRIM QE curve. Zero points for all magnitudes are calibrated by convolution with the Vega spectrum of Hayes \&
Latham (1975), renormalized (by a factor of 1.028) to zero magnitude. As a check on our synthetic photometry, we tabulated synthetic colors derived from the spectrophotometric atlas of Bruzual, Persson, Gunn, \& Stryker (BPGS; Gunn \& Stryker 1983) which includes infrared (IR) distributions from Strecker, Erickson, \& Witteborn (1979). Our synthetic colors agree with published colors for the corresponding spectral types to better than about $0.05 \mathrm{mag}$ for blue spectral types (Zombeck 1990). 
We predict colors for each program WD star from its best-fit gravity and temperature using a two-dimensional linear interpolation between the colors derived for the nearest grid models. Predicted colors for each program star are combined with observed visual DA magnitudes, to predict their $J$ and $K$ magnitudes, assuming that they are isolated. A table of $J_{p}$ and $K_{p}$ (predicted $J$ and $K$ magnitudes) for DA stars are listed in Table 4 along with the observed magnitudes.

\subsection{Deriving Distances and Searching for IR Excess}

Because each of the model spectra are presented in stellar surface fluxes $F_{\lambda}$ (in cgs units ergs $\mathrm{cm}^{-2} \mathrm{~s}^{-1} \mathrm{~cm}^{-1}$ ), magnitudes we derive for each model in the NLTE grid are effectively unnormalized surface magnitudes $V_{s}=2.5 \log F_{\lambda}$. The formula we derive for converting the best fit DA WD surface fluxes $F_{\lambda}$ (in ergs $\mathrm{cm}^{-2} \mathrm{~s}^{-1} \mathrm{~cm}^{-1}$ ), surface gravities $\log g$, and masses $M / M_{\odot}$ into absolute magnitude is

$M_{V}=-2.5 \log F_{\lambda}+2.5 \log g-2.5 \log \left(\frac{M}{M_{\odot}}\right)+31.042$.

Our own resulting values of $M_{V}$ differ little from those for 35 DAs in common with Vennes et al. 1997b (mean difference $0.01 \pm 0.24 \mathrm{mag}$ ). Since $M_{V}$ correlates strongly with $\log g$, this is consistent with the more detailed comparison performed by NGS99 of internal, external, and systematic errors in $\log g$ and $T_{\text {eff }}$ between the two studies. For distances to the DA WD, we compare to tabulated $V$ magnitudes, mostly from Table 3 of Marsh et al. (1997).

We derive the $K$ magnitude of any cool companion, $K_{c}$, from the difference between the predicted magnitude of the DA WD, $K_{p}$, and the total observed magnitude $K_{o}$ using

$$
K_{c}=K_{p}-2.5 \log \left[\operatorname{dex} 0.4\left(K_{p}-K_{o}\right)-1\right] \text {. }
$$

In Table 5 we list the properties of candidate cool companions detected in our IR-observed sample of DA WDs, esti- mated using the combination of the best-fit NLTE models of NGS99, our own infrared photometry, and published optical magnitudes. Column (1) lists REJ name. The companion $J$ and $K$ magnitudes calculated as detailed above are listed in columns (2) and (3). Spectral type of the candidate companions can be crudely estimated from the derived $M_{K_{c}}$ or from $J-K$. Since the combined errors on $M_{K_{c}}$ are in general lower, we list this in column (4) and the resulting spectral type estimates in columns (5) and (6), assuming young or old disk population for the red dwarf. The implied $V$ magnitude (from $V-K$ for the old disk spectral type) is shown in column (7), with notes in column (8).

\subsection{Candidate Cool Companions}

Our adopted criteria for claiming a significant near-IR excess over the predicted values for an isolated DA is simply a $3 \sigma$ excess in both $J$ and $K$ relative to the predicted IR magnitudes of the DA white dwarf, $J_{p}$ and $K_{p}$. Errors in our observed $K$ magnitudes are generally 2-3 times higher than for $J$, but provide greater wavelength contrast to the optical. We do not use $(J-K)$ as a discriminant because its combined errors are highest. The errors we adopt for the excesses $\left(J_{p}-J_{o}\right)$ and $\left(K_{p}-K_{o}\right)$ include our photometric errors in $J$ and $K$, combined with assumed errors of $0.1 \mathrm{mag}$ each on the predicted values of $K_{p}$ and $J_{p}$.

As an example, for REJ $1845+682$, we calculate from the published $V$ magnitude and model atmosphere fits in Table 1 a predicted magnitude with its estimated error of $K_{p}=$ $16.2 \pm 0.1$ (Table 4). The difference between this and the observed magnitude $K=14.37 \pm 0.19$ (Table 4) is thus $K_{\text {excess }}=1.83 \pm 0.21 \mathrm{mag}$, qualifying as an $8 \sigma$ excess.

Of the 47 objects with reliable $K$ photometry (which excludes REJ $1235+233 /$ PG $1232+238$ ) we find 10 systems with a significant IR excess (see Fig. 2). Of these, five are known binary systems. Of the five that are newly recognized systems with IR excess, four have spatially resolved objects discernible either in the near-IR or optical images within

TABLE 5

Derived Properties of Candidate Cool Companions

\begin{tabular}{|c|c|c|c|c|c|c|c|}
\hline \multirow[b]{2}{*}{$\begin{array}{l}\text { REJ NAME } \\
\text { (1) }\end{array}$} & \multirow[b]{2}{*}{$\begin{array}{l}J_{c} \\
(2)\end{array}$} & \multirow[b]{2}{*}{$\begin{array}{l}K_{c} \\
(3)\end{array}$} & \multirow[b]{2}{*}{$\begin{array}{c}M_{\boldsymbol{K}_{c}} \\
\text { (4) }\end{array}$} & \multicolumn{2}{|c|}{ M SUBTYPE } & \multirow{2}{*}{$\frac{V_{c}^{\mathbf{b}}}{(\mathrm{OD})}$} & \multirow[b]{2}{*}{$\begin{array}{c}\text { Notes } \\
(8)\end{array}$} \\
\hline & & & & $\begin{array}{c}\text { (YD) } \\
(5)\end{array}$ & $\begin{array}{c}\text { (OD) } \\
(6)\end{array}$ & & \\
\hline $0134-160 \ldots \ldots$ & 13.3 & 12.3 & 7.4 & 4.5 & 3.5 & 17.4 & 1 \\
\hline $0148-253 \ldots \ldots$ & 12.4 & 11.6 & 6.9 & 4 & 3 & 16.4 & 1 \\
\hline $0427+740 \ldots \ldots$ & 16.4 & 16.2 & 9.4 & 6.5 & 6 & 23.5 & 3 \\
\hline $0916-194 \ldots \ldots$ & 14.0 & 13.7 & 7.7 & 5 & 4 & 19.2 & \\
\hline $1036+460 \ldots \ldots$ & 12.8 & 12.0 & 7.6 & 5 & 4 & 17.3 & 1 \\
\hline $1126+183 \ldots \ldots$ & 13.0 & 12.1 & 6.5 & 3.5 & 2.5 & 16.6 & 1 \\
\hline $1629+780 \ldots \ldots$ & 11.1 & 11.3 & 7.7 & 5 & 4 & 16.6 & 1 \\
\hline $1711+664 \ldots \ldots$ & 15.1 & 14.2 & 8.0 & 5.5 & 5 & 20.0 & 2 \\
\hline $1820+580 \ldots \ldots$ & 15.5 & 13.8 & 8.9 & 6 & 5.5 & 21.0 & \\
\hline $1845+682 \ldots \ldots$ & 15.3 & 14.6 & 9.2 & 6.5 & 6 & 21.9 & \\
\hline
\end{tabular}

Notes.-(1) Known binary; see Table 1. (2) IR photometry known to include nearby marginally optically resolved object at separation indicated in Table 1. (3) This star is spatially resolved at near-IR wavelengths, with a companion within less than 2". Some nebulosity (diffuse emission) is also noted nearby. The more distant companion noted in Table 1 is not included in the IR photometry.

${ }^{a}$ Approximate spectral type from $M_{K_{c}}$ and Leggett 1992, Table 6, for Young Disk and Old Disk stars.

${ }^{\mathrm{b}}$ Approximate $V$ magnitude of companion assuming Old Disk population, from $M_{K_{c}}$ and $(V-K)$ of Leggett 1992, Table 6. 

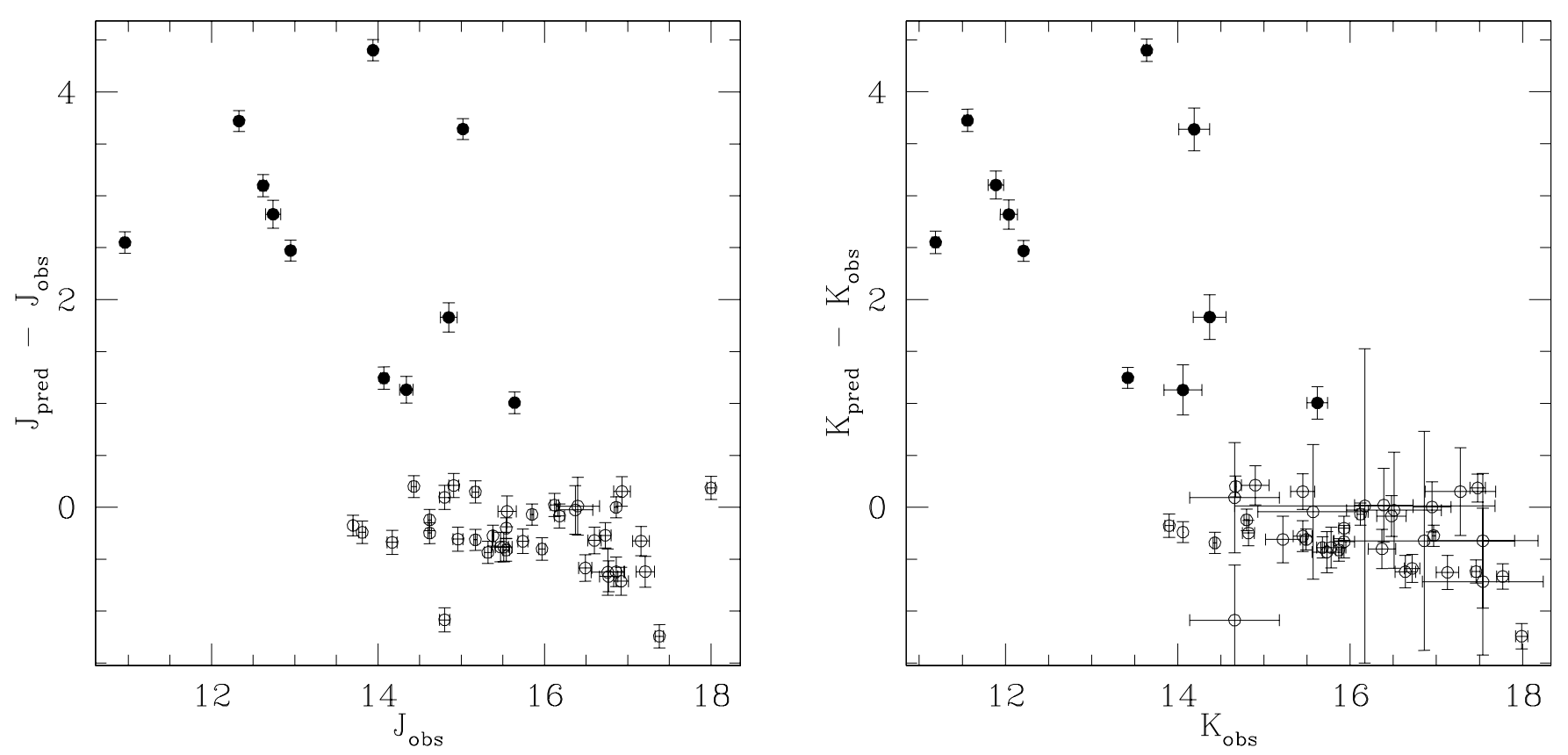

FIG. 2.-Difference between predicted and observed magnitudes plotted against observed magnitude for IR-observed hot DA white dwarfs. Objects for which we determine a significant IR excess are shown as filled circles.

$10^{\prime \prime}$. In only some cases, noted in Table 5 , these objects are included in the near-IR photometry.

The overall properties of DAs with IR excess reveal no obvious bias within our observed sample. The mean distance of the DAs without excess is $144 \pm 98 \mathrm{pc}$, while those 11 with excess have $117 \pm 53 \mathrm{pc}$. Similarly, the mean values and distributions of $T_{\text {eff }}, \log g, V$, and $M_{V}$ of the DAs without excess are indistinguishable from those 11 with excess.

The mean value of $\left(K_{p}-K_{o}\right)$ for the systems with no IR excess is $-0.28 \pm 0.33$. We find a similar offset for $\left(J_{p}-J_{o}\right)$ of $-0.30 \pm 0.35$. $^{4} \mathrm{We}$ consider several possibilities to explain the zero point offset. (1) Our predicted $D A(V-K)$ colors are too red. Since we derive our DA $K$ magnitudes from the predicted color $(V-K)_{p}$ and the published $V$ magnitude, a difference in normalization in our synthetic $V$ or $K$ photometry of the DA models could cause the offset. However, the synthetic $V$ and $K$ magnitude and colors we derive for stars in the BPGS spectral library match their normalizations and colors for each spectral type (see $\S 4.1$ above), and our $(V-K)$ colors also match those in the spectral library of Pickles (1998) to within a mean difference of $0.01 \pm 0.1$ mag. (2) Our IR photometric zero point is too faint. Comparing the two DAs also observed by Zuckerman \& Becklin (1992), our $K$ magnitude are 0.1 mag fainter in each case. While this difference is well within our errors, we cannot rule out a photometric zero point shift of this order as a contributor to the mean zero point offset. (3) Unmodeled, large equivalent width IR absorption features in DAs. To account for a shift of $0.3 \mathrm{mag}$ in $K$, spectral absorption features with equivalent widths of nearly $1440 \AA$ would be required that are not present in the DA models. Given that different absorption features would be required

\footnotetext{
${ }^{4}$ The mean of $(J-K)_{p}-(J-K)_{o}$ is zero $(-0.03 \pm 0.29)$.
}

in the $J$ band to yield a similar zero point shift, we disregard this possibility.

Even with this unexplained zero point shift, our determination of an IR excess, based on the significance of $\left(K_{p}-K_{o}\right)$, are unaffected. However, our estimated companion magnitudes $K_{c}$ and absolute magnitudes $M_{K_{c}}$, may be up to $0.3 \mathrm{mag}$ too faint, leading in turn to spectral subclass estimates approximately half a subclass too late.

The $V$ magnitudes of the candidate companions listed in Table 5 are in most cases significantly fainter than the visual magnitudes of the DA WDs. Since we elected not to observe in the IR those systems known to be composite by their optical spectra, this result is expected from our selection.

\subsection{Spatially Resolved Companions}

Finding charts for most of our sample have been published by Shara et al. (Shara, Shara, \& McLean 1993; Shara et al. 1997). To account for visually resolved companions, we inspected images from the DSS (Shara et al. 1997) and also searched the USNOA-1.0 (Monet 1996) catalog to $R \sim 19.5$ for all stars within $15^{\prime \prime}$, of the program star. Resolved stars within a maximum distance of $10^{\prime \prime}$, and within $3 \mathrm{mag}$ (in $R$ ) of the primary are noted in Table 1 as visual pairs. For the closest WD distances in our sample of $\sim 20 \mathrm{pc}$, the spatial resolution of $\sim 2^{\prime \prime}$ in both the archival optical and new IR images, corresponds to $40 \mathrm{AU}$. On the other hand, a search limited to $10^{\prime \prime}$, corresponds to maximum separations of $200 \mathrm{AU}$. For the farthest WDs in our sample near $500 \mathrm{pc}$, imaging is instead sensitive to resolved companions between 1000 and $5000 \mathrm{AU}$.

\section{NOTES ON INDIVIDUAL OBJECTS}

REJ 0134-160.-Based on radial velocity measurements (Schultz, Zuckerman, \& Becklin 1996) and its lowmass (NGS99; Finley, Koester, \& Basri 1997) mass transfer 
is unlikely to have occurred in this binary DA $+M$ star system.

REJ 0148 - 253.-Finley et al. (1997) claimed evidence for a companion based on $\mathrm{H} \beta$ emission and a slight red excess. Our result of an IR excess confirms the presence of the companion.

REJ $0427+7406$. - This star appears to be a multiple at near-IR wavelengths. Some nebulosity (diffuse emission) is also noted nearby. A finding chart is provided in Figure 3.

REJ 0443-034.-This is the hottest DA in our sample of IR-observed WDs. DA stars with such high temperatures should cool extremely rapidly and thus be detected only rarely. The suggestion by Finley et al. (1997) of a possible red excess is not borne out by our measurements.

REJ 0512-004.- As the least massive DA in the sample, this DA might be expected to have a companion, since very low-mass WDs are thought to form in binaries (Marsh, Dhillon, \& Duck 1995). The absence of IR excess indicates a very cool and/or degenerate companion at best. Our IR photometric method is insensitive to such objects, which are best detected through radial velocity variations (Saffer, Livio, \& Yungelson 1998). We discuss the overall effect on our estimated binary fraction in $\S 7$.

REJ 0916-194.- -While its IR excess suggests a late-type companion, our estimate for its visual magnitude $V_{c}$ is only $\sim 2$ mag fainter than the total $V$ magnitude, so evidence for the companion should have been seen in the red end of its optical spectrum. However, the only spectrum (from Vennes et al. 1997b) is of comparatively low S/N and extends only to $6040 \AA$. We expect that a higher $\mathrm{S} / \mathrm{N}$ optical spectrum extending farther redward, or our own planned IR spectroscopy will readily reveal the companion.

$R E J 1036+460(P G 1033+464)$. $-K$ photometry and $K$ excess from Zuckerman \& Becklin (1992) are within $0.1 \mathrm{mag}$ of the values we report here. However, their derived absol-

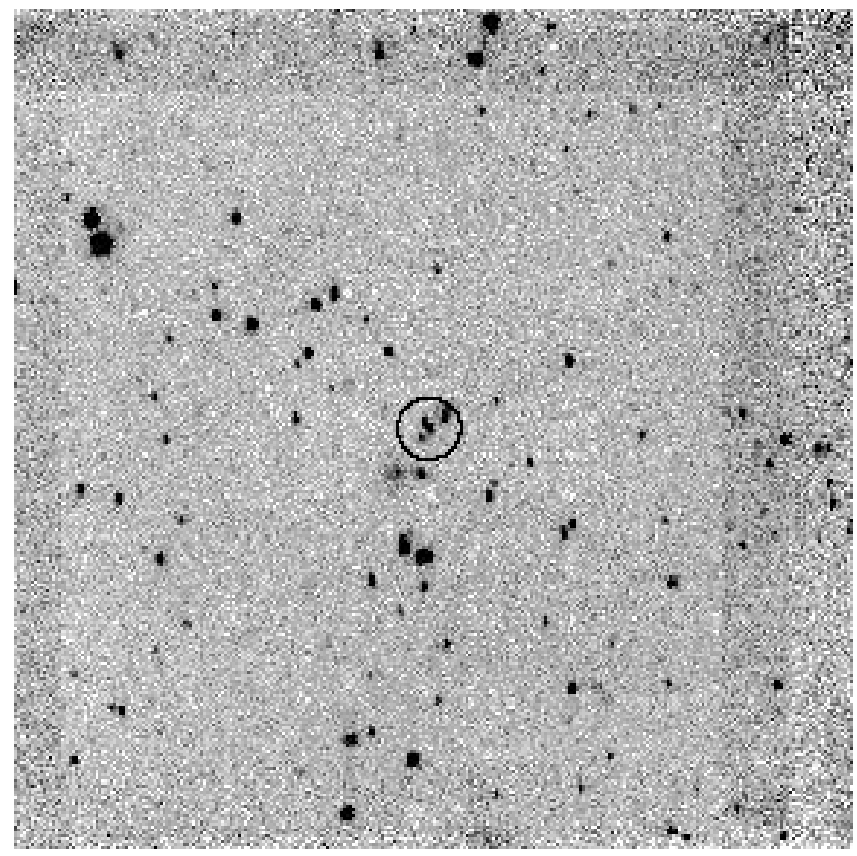

FIG. 3. $-5^{\prime} \times 5^{\prime} K$-band image of the field of REJ $0427+7406$ reveals several close neighbors to the DA. Diffuse emission is also evident to the SE, possible a background galaxy. North is up, east to the left. ute red dwarf magnitude $M_{\mathbf{K}_{c}}$ is fainter by $1.5 \mathrm{mag}$. Rather than using detailed spectral fits, they had to use published color-estimated distance moduli that assumed uniform surface gravity for all white dwarfs, while in fact the absolute magnitude depends strongly on $\log g$. For the other object in common between our samples, REJ $1126+183$ (PG 1123 + 189), we note an even more striking discrepancy (3.2 mag in $M_{K_{c}}$ ). They assume a distance of $30 \mathrm{pc}$, while the best estimate from detailed fits of NGS is $135 \mathrm{pc}$. Their resulting claim that the IMF is flat or increasing down to at least $0.1 M_{\odot}$ should be revisited with more accurate distance determinations.

REJ $1043+490$. - This was suggested by Schwartz et al. (1995) as a possible multiple system based on a slightly red $R-I$ color, but they suggested that further measurements were needed to confirm this. The residuals of the DA model fit to the optical spectrum in NGS99 confirmed a red excess for $\lambda>4500 \AA$ corresponding to a companion with $V \sim 18.2$ or $M_{V} \sim 10.4$ at our distance of $370 \mathrm{pc}$. However, our near-IR photometry is inconsistent with such a companion, since a normal late $M$ dwarf companion of this absolute magnitude and distance would yield $J \sim 14.5$ and $K \sim 13.6$. We note that the system is a visual binary, with a red companion $8^{\prime \prime}$ West of the DA. Contamination of the DA spectrum in NGS99 is thus the likely explanation.

REJ 1235+233 (PG1232+238). - The object is barely discernible in our $K$ mosaic, so reported $K$ photometry is poor.

REJ $1711+664$. - This is a barely resolved visual pair, with a late-type star only $\sim 2$ ".5 distant, which is included in our IR photometry (Mason et al. 1995). Jomaron (1997) also found the M-type secondary in residuals to the DA optical spectrum, classifying the former as type M3-M4, with no apparent $\mathrm{H}$ emission. Our IR photometry indicates a somewhat later type.

REJ $2207+252$. - IR photometry for $2207+252$ was contaminated by a very bright, resolved object at 8.9.

\section{SELECTION BIASES}

Strong biases are likely to be inherent in any sample of EUV-detected WDs. The strongest is the selection against a high column of intervening interstellar material. Columns as low as $5 \times 10^{19} \mathrm{~cm}^{-2}$ easily quench flux in the EUV below detection levels achieved in either the ROSAT WFC or the $E U V E .{ }^{5}$ When compared to more distant areas in the Galactic plane, the local interstellar medium lacks cool dense material $\left(n \sim 0.1 \mathrm{~cm}^{-3}, T \sim 100 \mathrm{~K}\right)$. This "local bubble" has a mean radius of 70 pc (Diamond, Jewell, \& Ponman 1995). Accurate estimates of the intervening column to each star are difficult, requiring high S/N EUV spectral analysis well beyond the scope of this work. The global effect amounts to a direction-dependent flux sensitivity limit (and therefore volume) that is strongly dependent on the fine structure of the ISM.

As pointed out by NGS99, at a given temperature, WDs with lower masses are larger, consequently more luminous, and thus detectable to larger distances. For instance, at $30,000 \mathrm{~K}$, a WD of $0.5 M_{\odot}$ is detectable at distances $30 \%$

\footnotetext{
${ }^{5}$ For example, one optical depth is reached at column densities of 30 , 5,1 , and $0.5 \times 10^{18} \mathrm{~cm}^{-2}$, respectively, for the EUVE $100,200,400$, and $600 \AA$ A detection bandpasses (Vennes et al. 1996).
} 


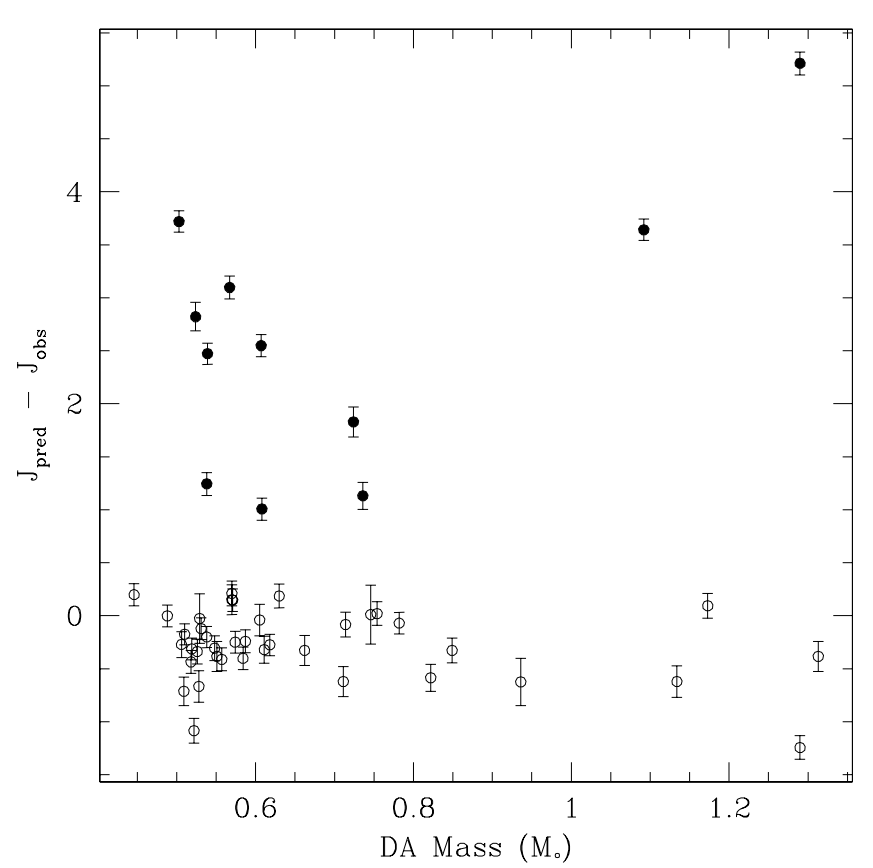

FIG. 4.- $J$ excess (difference between predicted and observed $J$ magnitudes) plotted against DA white dwarf mass in solar units from the best-fit model atmospheres of NGS99. We see no evidence that IR excess correlates with DA mass.

larger than a $0.7 M_{\odot}$ WD. For many WDs at the maximum distance sampled by most optical surveys, interstellar matter effectively absorbs all EUV radiation. Thus, the dominant effect in EUV surveys is probably not a selection for massive WDs as suggested by Vennes et al., but a selection against low-mass WDs due to a sample volume strongly affected by interstellar absorption. However, there is no direct bias induced by interstellar absorption on the observed binary fraction.

There have been suggestions that detection of soft X-ray flux from hot white dwarfs may be biased against close binary systems. This could arise if heavy elements arising in a wind from the MS companion have accreted onto the surface of the white dwarf, enhancing atmospheric EUV and soft X-ray opacity (Barstow et al. 1993). By including only DA stars in our sample, we might thus be preferentially excluding some binary systems. For instance, the hot white dwarf in the close binary RE $1016-053$ is a DAO star whose mixed $\mathrm{H} / \mathrm{He}$ composition is likely to be attributable to slow accretion from a red dwarf companion (Vennes et al. 1997a). However, Barstow et al. (1994) found that hot ROSAT (PSPC or EUV) detected WDs in noninteracting binaries with early-type stars showed no signs of abundance enhancements from accretion, their opacity sources following the same temperature pattern as seen for isolated WDs. Indeed, while those abundance enhancements may lead to significant X-ray opacity (e.g., Dupuis et al. 1995) in the hottest WDs, they remain strong EUV sources, particularly at $\lambda>200 \AA$.

Arguments for biases of opposite effect exist as well- that increased EUV flux is more likely from a WD that suffers reheating due to accretion of material from its companion. Since the DAs in our sample are not significantly variable, the interaction would have to be fairly weak or intermittent (e.g., via weak wind accretion). Furthermore, Barstow et al.
(1992) find for the DA + K2V binary system V471 Tauri that the temperature change in such cases (with accretion rates of $0.4-11 \times 10^{-13} M_{\odot} \mathrm{yr}^{-1}$ ) is small and occurs only in the region near the hot spot of the accretion column.

Measurement of these putative competing selection effects would clearly benefit from larger samples. A large number of EUV-detected DA white dwarfs with good constraints from atmospheric modeling now exist (Marsh et al. 1997; Vennes et al. 1997b; Finley, Koester, \& Basri 1997; NGS99). The 2MASS will provide (less sensitive, $K \leq 14$ ) IR photometry for brighter subsamples.

WDs with masses less than the canonical core-helium ignition mass $\left(0.49 M_{\odot}\right.$; Sweigart 1994) are likely to be in short-period binary systems, where their evolution was cut short by wind ejection during a common envelope phase (Marsh et al. 1995; Marsh 1995). Only one of the WD stars in our sample, REJ $0512-004$, has a low mass $\left(0.45 M_{\odot}\right)$, but it shows no signs of a companion. On the other hand, lower mass companions may be preferentially removed by merging, a process that is more effective the more massive the WD.

It is thus of interest to check for any correlation between DA mass and IR excess. Figure 4 shows that no such correlation is detectable in our sample, as confirmed by two correlation tests. Similarly, there is no correlation between the absolute $K$ magnitudes of companions $M_{K_{C}}$ and those of their corresponding DA white dwarfs. ${ }^{6}$ Two sample statistical tests also reveal no significant difference between the mass or absolute magnitude distributions of the DAs in the no-excess and excess subsamples. ${ }^{7}$ Larger samples would put better constraints on these hypotheses.

\section{THE BINARY FRACTION}

Our WD sample demands that the initial primary has already passed through the AGB phase, imposing a lower mass limit of about $2 M_{\odot}$, on the WD progenitor. We thus probe portions of the (initial) mass ratio distribution that are quite difficult to reach in unevolved systems. The binary frequency of high-mass stars has proven difficult to determine for several reasons. Photometric detection of a faint low-mass star next to a bright early type primary requires high precision photometry. The use of radial velocity variations can also be difficult due to the large rotational speeds of many early-type stars - one reason why solar-type stars are preferred in current planetary searches.

Out of our uniformly selected sample of 57 EUV-detected DA white dwarfs, including our new detections of IR excess, we find strong evidence for multiplicity in 20 systems (35\%) within a $10^{\prime \prime}$, radius. Simple Poisson statistics on the subsample yields an error of $7 \%$. We may also count multiple systems by assuming that any resolved object within the $10^{\prime \prime}$, radius is at the same distance as the WD (i.e., a presumed companion), and thereby tally objects within a chosen limiting physical separation (projected onto the sky

\footnotetext{
${ }^{6}$ Generalized Kendall's Tau and Cox Proportional Hazard model tests from the ASURV package (LaValley et al. 1992) show probabilities $P$ of $18 \%$ and $72 \%$, respectively, that a correlation is not present between $\left(J_{p}-J_{o}\right)$ and DA mass. A significant correlation requires $P>99 \%$. $P$ of $26 \%$ and $36 \%$, respectively, are obtained for $M_{K_{c}}$ vs. $M_{V_{\mathrm{DA}}}$.

${ }^{7}$ Generalized Wilcoxon and logrank tests show that the two samples are inconsistent with being drawn from the same parent population at only the $P \sim 75 \%$ level for DA mass and only the $P \sim 93 \%$ level for $M_{V_{\mathrm{DA}}}$.
} 
plane). Since the largest estimated distance to any of the WDs in our sample is 470 pc (for REJ 1235+233) we choose for completeness ${ }^{8}$ a maximum projected physical separation of $1000 \mathrm{AU}$. This removes only one system $(1726+583)$, leaving the binary fraction essentially unchanged at $33 \pm 7 \%$.

For comparison, among a complete sample of nearby $(d<5$ pc) M dwarfs, Leinert et al. (1997) found $26 \pm 9 \%$ to have companions. In a meta-analysis, Fischer \& Marcy (1992) found that $42 \pm 9 \%$ of $\mathrm{M}$ dwarfs have main-sequence companions. This is lower than the best incompletenesscorrected estimate for $\mathrm{G}$ dwarfs ( $\sim 57 \%$; Duquennoy \& Mayor 1991), due in part to the smaller range of secondary companion masses available.

As allowed by the faintness of WDs relative to mainsequence companions, and the use of IR photometry, the current study is sensitive to a wide range of companion masses, from late $\mathrm{M}$ to early A type. The late-type companion masses corresponding to our estimates of absolute $K$ magnitudes $M_{K_{C}}$ range from about 0.5 down to $0.1 M_{\odot} \cdot{ }^{9}$ Judging by known or estimated spectral types of unresolved companions and by derived absolute magnitudes of the presumed companions discussed above, $90 \%$ of companions from Tables 1 and 5 are of spectral type $\mathrm{K}$ or later.

We note that despite the significant improvement in sensitivity to low-mass companions that our IR photometry affords, the binary frequency of our sample remains a lower limit for several reasons. First, very low-mass companions can fall below our IR detection limit of $K \sim 16$, with sensitivity decreasing with distance. Also, while we assume only a single degenerate in each system, double degenerates would not likely be distinguished by our IR photometry. Saffer et al. (1998) derive a fraction of double degenerate systems that may be as high as $20 \%$ when they include an estimated correction factor of $\sim 1.4$ for selection efficiency in their sample.

Some of the hot DAs with masses higher than expected from isolated evolution $\left(\sim 1.1 M_{\odot}\right)$ have been hypothesized to represent a population of coalesced double degenerate systems (e.g., Marsh et al. 1997). Such mergers would slightly reduce the observed binary fraction in our sample. While they might also help constrain the Type Ia supernova formation rate (e.g., Branch et al. 1995), the predicted peak of such a merger distribution should be near $0.9 M_{\odot}$, which is not seen.

\footnotetext{
${ }^{8}$ Assumes a spatial resolution of 2", at a similar maximum distance of 500 pc. Including only unresolved systems is intuitively appealing, but it introduces a bias against companions in nearby systems.

${ }^{9}$ Below about $0.2 M_{\odot}$, absolute magnitudes no longer correlate well with mass. There the relationship steepens considerably, in both $V$ and $K$ bands, and differs little as a function of metallicity (Baraffe et al. 1998; Henry et al. 1999).
}

Among our sample of hot DAs may be some close binaries that have passed through a phase of common envelope evolution. While these are sometimes called precataclysmic binaries, many will not become cataclysmic variables within a Hubble time (Ritter 1986). Based on morphological classification of planetary nebulae, and the assumption that axisymmetrical mass loss is caused by binary companions, Soker (1997) estimated that only $10 \%$ of PN had no interaction with any companion, stellar or substellar. Only $11 \%$ had interactions while avoiding a common envelope phase. For the case of close binaries, the observed distribution of companion masses may thus have been modified by evolution. Some systems may have coalesced, while others may have grown through wind accretion, or by stable mass transfer. As the progenitors of novae, CVs, symbiotics, Feige 24-type systems, and the intriguing dwarf carbon (dC) stars (deKool \& Green 1995), these WD + MS systems warrant the improved constraints on their masses and abundance enhancements that IR spectroscopy of our sample will provide. For instance, the dC stars, which likely evolve into $\mathrm{CH}$ or $\mathrm{Ba}$ giants, are dwarfs whose strong carbon bands betray episodes of masstransfer from the previously AGB companion, now the WD (Green 2000). There is recent evidence (Heber et al. 1993; Liebert et al. 1994) that dCs may show stronger abundance enhancements soon after the AGB phase, i.e., while the WD is still hot. IR spectra would test for this, and its potential ramifications for the depths and timescales of mixing due to chemical potential gradients (e.g., Proffit \& Michaud 1989).

We are obtaining IR spectra of these systems at the KPNO $2.1 \mathrm{~m}$ with CRSP to better constrain the mass and spectral type of the cool companions presumably causing the IR excess. We await these results before pursuing more detailed discussions of the mass function of the companions, the observed final and implied initial mass ratios of the systems, and detailed simulations of the effects of binary evolution. The binary fraction may be a function of the mass ratio, or of the primary mass; these fundamental questions of stellar evolution remain open. If the observed binary fraction for DA WDs proves to be inconsistent with those of main-sequence primaries in a similar (initial) mass range, it could mean that age-dependent formation conditions (e.g., abundance, kinematics, or turbulence) strongly influence the binary fraction.

Many thanks to Dick Joyce and the staff at KPNO for all their help during IRIM observing runs. Thanks to Fred Ringwald for providing a catalog of unresolved, hot-highgravity with cool companion composites. The author gratefully acknowledges support provided by NASA through grant NAG5-1253, and contract NAS8-39073 (CXC). This research has made extensive use of SIMBAD.

\section{APPENDIX}

\section{PHOTOMETRY OF MOSAICKED IMAGES}

For some targets, visual inspection failed to reveal the source on the individual images. We derive photometry these targets from a global mosaic of the field produced by shifting and combining individual frames. We use integer pixel shifts and average the overlap regions between frames, accounting for background/sky variations by subtracting the median value of the frame from itself before averaging the fields. Integer pixel shifts do not necessarily conserve flux but are employed here for two 
reasons: (1) the stellar images on our frames are sub-Nyquist sampled. (2) The presence of significant dead (insensitive) space between pixels. The undersampled profiles of the star generally ensure that most of the stellar flux is contained within one pixel, and the presence of dead space combined with undersampled images makes it difficult to produce flux conserved stellar profiles in the first place. Thus, we conclude that mosaicking images will not significantly alter our photometry. These assumptions are tested by comparing magnitudes derived from a mosaicked image to those from individual frames. Figure 5 illustrates this comparison. Figure 5 demonstrates that photometry from mosaicked images is consistent (within the errors) with photometry obtained from individual frames.

The mosaicked images, however, only allow us to make an estimate of the formal (Poisson + detector noise) errors and do not account for any systematic errors or any additional possible degradation of the $\mathrm{S} / \mathrm{N}$ created by the photometry software. A comparison of the formal errors with those obtained by comparing the rms magnitude variation from individual frame suggests that our errors are dominated by these systematic errors. The dashed line in Figure 6 shows the magnitude of the formal errors. The solid dots are the observed rms variations of magnitudes from individual frames. Clearly, formal errors underestimate the measured empirical estimates. The formal errors are derived using a modified form of the IRAF task

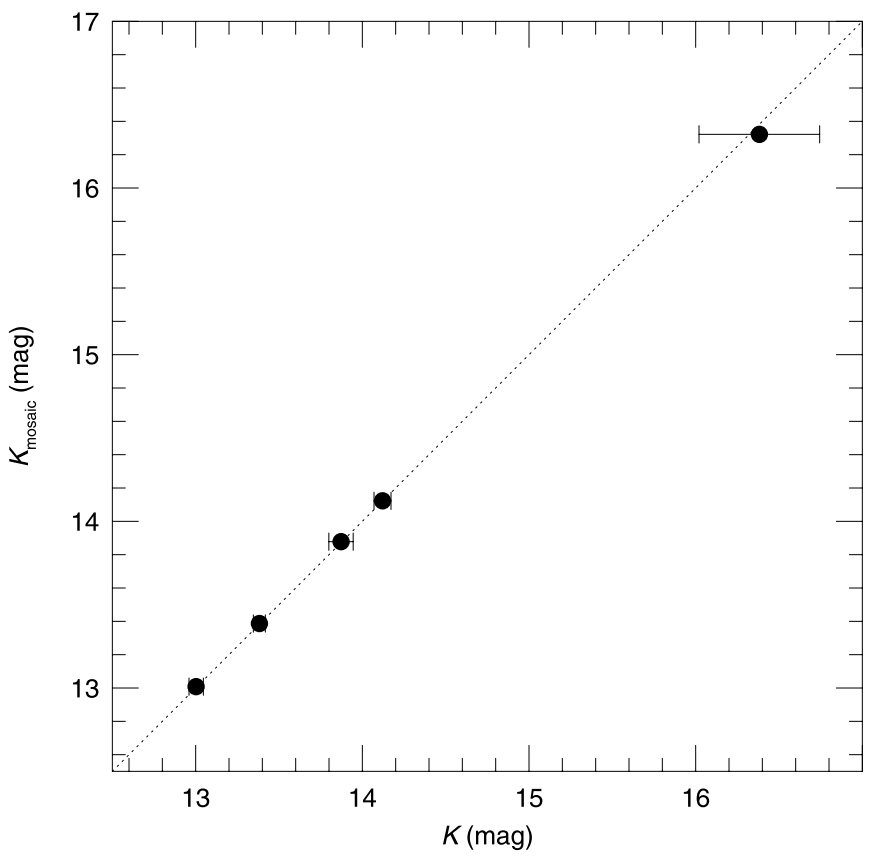

FIG. 5.-Comparison of the derived magnitudes in the field of PG $0904+511$ using individual frames and a mosaic image. $K$-band magnitudes derived from an image mosaic and those from individual frames are compared for five stars in the PG $0904+511$ field. The error bars show the $1 \sigma$ deviation among magnitudes from individual frames. The dashed line shows the resulting relationship if the two quantities were identical.

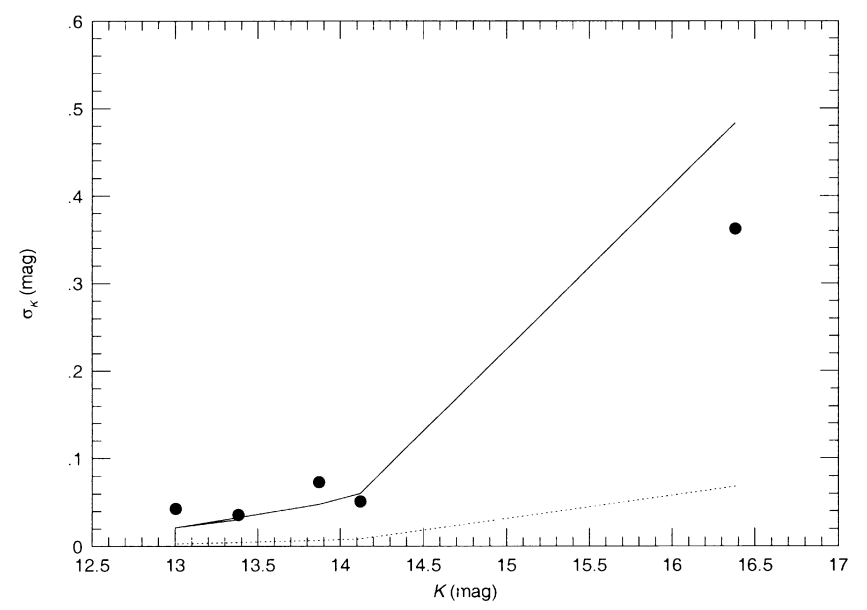

FIG. 6.-Observed error (estimated as the $1 \sigma$ deviation among magnitudes derived from individual frames) vs. magnitude for the PG $0904+511$ field. The dashed line shows the magnitude of the formal errors from $\mathrm{S} / \mathrm{N}$ arguments. The solid line shows our error curve for the field (see text for details). These data suggest that we are dominated by systematic errors. 
"ccdtime" (modified to run on the IDL package). To obtain a realistic estimate for the errors on mosaicked images, we modeled the effects of systematic errors by artificially increasing the readout noise of the detector and minimizing the relationship:

$$
\sum_{i=1}^{N} \frac{\left(\sigma_{\mathrm{obs}}-\sigma_{\mathrm{cal}}\right)^{2}}{\sigma_{\mathrm{obs}}^{2}},
$$

where $\sigma_{\mathrm{obs}}$ are the observed errors, $\sigma_{\mathrm{cal}}$ are errors calculated by increasing readout noise, and $N$ is the number of stars in this experiment. The solid line in Figure 6 shows an example of this fit. We use the best-fit "readout noise" to extrapolate the formal $\mathrm{S} / \mathrm{N}$ to stars for which only mosaicked image photometry is available.

\section{REFERENCES}

Baraffe, I., Chabrier, G., Allard, F., \& Hauschildt, P. H. 1998, A\&A, 337, 403

Barstow, M. A., et al. 1992, MNRAS, 255, 369

Barstow, M. A., et al. 1993, MNRAS, 264, 16

Barstow, M. A., et al. 1994, MNRAS, 270, 499

Burleigh, M. R., Barstow, M. A., \& Fleming, T. A. 1997, MNRAS, 287, 381

Branch, D., Livio, M., Yungelson, L. R., Boffi, F. R., \& Baron, E. 1995, PASP, 107, 1019

Cash, W., Charles, P., \& Johnson, H. M. 1980, ApJ, 239, L23

Casali, M. M., \& Hawarden, T. G. 1992, JCMT-UKIRT Newsletter, 3, 33

Catalàn, M. S., Sarna, M. J., Jomaron, C. M., \& Cannon, S. R. 1995, MNRAS, 275, 153

deKool, M., \& Green, P. J. 1995, ApJ, 449, 236

deKool, M., \& Ritter, H. 1993, A\&A, 267, 397

Diamond, C. J., Jewell, S. J., \& Ponman, T. J. 1995, MNRAS, 274, 589

Dreizler, S., Heber, U., Jordan, S., \& Engels, D. 1994, in Hot Stars in the Galactic Halo, ed. S. J. Adelman, A. R. Upgren, \& C. J. Adelman (Cambridge: Cambridge Univ. Press), 228

Dupuis, J., Vennes, S., Bowyer, S., Pradhan, A. K., \& Thejll, P. 1995, ApJ, 455,574

Duquennoy, A., \& Mayor, M. 1991, A\&A, 248, 485

Finley, D. S., et al. 1993, ApJ, 417, 259

Finley, D. S., Koester, D., \& Basri, G. 1997, ApJ, 488, 375

Fischer, D. A., \& Marcy, G. W. 1992, ApJ, 396, 178

Fowler, A. M., Gatley, I., Stuart, F., Joyce, R. R., \& Probst, R. G. 1988, Proc. SPIE, 972, 107

Green, P. J. 2000, in Proc. IAU Symp. 177, The Carbon Star Phenomenon, ed. R. F. Wing (Dordrecht: Kluwer), in press

Green, P. J., \& Margon, B. 1994, ApJ, 423, 723

Green, R. F., Schmidt, M., \& Liebert, J. 1986, ApJS, 61, 305

Gunn, J. E., \& Stryker, L. L. 1983, ApJS, 52, 121

Hayes, D. S., \& Latham, D. W. 1975, ApJ, 197, 593

Heber, U., Bade, N., Jordan, S., \& Voges, W. 1993, A\&A, 267, L31

Henry, T. J., et al. 1999, ApJ, 512, 864

Howell, S. B. 1989, PASP, 101, 616

Jomaron, C. M. 1997, Ph.D. thesis, University College, London

LaValley, M., Isobe, T., \& Feigelson, E. D. 1992, in Astronomical Data

Analysis Software \& Systems, ed. D. Worrall et al. (San Francisco: ASP)

Leggett, S. K. 1992, ApJS, 82, 351

Leinert, C., Henry, T. J., Glindemann, A., \& McCarthy, D. W., Jr. 1997, A\&A, 325, 159

Liebert, J., et al. 1994, ApJ, 421, 733

Liebert, J., Bergeron, P., \& Saffer, R. A. 1990, PASP, 102, 1126

Malina, R., et al. 1994, AJ, 107, 751

Marsh, M. C., et al. 1997, MNRAS, 286, 369

Marsh, T. R. 1995, MNRAS, 275, 89

Marsh, T. R., Dhillon, V. S., \& Duck, S. R. 1995, MNRAS, 275, 828

Mason, K. O., et al. 1995, MNRAS, 274, 1194

McCook, G. P., \& Sion, E. M. 1999, ApJS, 121, 1

Monet, D. 1996, BAAS, 188, 5404

Napiwotzki, R., Green, P. J., \& Saffer, R. A. 1999, ApJ, 517, 399 (NGS99)

Pickles, A. J. 1998, PASP, 100, 863

Pounds, K., et al. 1993, MNRAS, 260, 77

Probst, R. 1983a, ApJS, 53, 335

.1983b, ApJ, 274, 237

Proffitt, C. R., \& Michaud, G. 1989, ApJ, 345, 998

Ritter, H. 1986, A\&A, 169, 139

Saffer, R. A., Livio, M., \& Yungelson, L. R. 1998, ApJ, 502, 394

Schmidt, G. D., Bergeron, P., Liebert, J., \& Saffer, R. A. 1992, ApJ, 394, 603

Schultz, G., Zuckerman, B., \& Becklin, E. E. 1996, ApJ, 460, 402

Schwartz, R. D., Dawkins, D., Findley, D., \& Chen, D. 1995, PASP, 107, 667

Shara, M. M., Bergeron, I. E., Christian, C. A., Craig, N., \& Bowyer, S. 1997, PASP, 109, 998

Shara, M. M., Shara, D. J., \& McLean, B. 1993, PASP, 105, 387

Soker, N. 1997, ApJS, 112, 487

Strecker, D. W., Erickson, E. F., \& Witteborn, F. C. 1979, ApJS, 41, 501

Sweigart, A. V. 1994, ApJ, 426, 612

Vennes, S., Christian, D. J., \& Thorstensen, J. R. 1998, ApJ, 502, 763

Vennes, S., Dupuis, J., Bowyer, S., \& Pradhan, A. K. 1997a, ApJ, 482, L73

Vennes, S., Thejll, P. A., Wickramasinghe, D. T., \& Bessell, M. S. 1996, ApJ, 467, 782

Vennes, S., Thejll, P. A., Galvan, R. G., \& Dupuis, J. 1997b, ApJ, 480, 714

Vennes, S., \& Thorstensen, J. R. 1994, ApJ, 433, L29

Wonnacott, D., Kellett, B. J., \& Stickland, D. J. 1993, MNRAS, 262, 277

Zombeck, M. V. 1990, Handbook of Space Astronomy and Astrophysics (Cambridge: Cambridge Univ. Press)

Zuckerman, B., \& Becklin, E. 1992, ApJ, 386, 260 